TRANSACTIONS OF THE

AMERICAN MATHEMATICAL SOCIETY

Volume 362, Number 1, January 2010, Pages 319-344

S 0002-9947(09)04877-6

Article electronically published on July 27, 2009

\title{
FINSLER GEOMETRY AND ACTIONS OF THE $p$-SCHATTEN UNITARY GROUPS
}

\author{
ESTEBAN ANDRUCHOW, GABRIEL LAROTONDA, AND LÁZARO RECHT
}

In memory of A. R. Larotonda (1939-2005)

\begin{abstract}
Let $p$ be an even positive integer and $U_{p}(\mathcal{H})$ the Banach-Lie group of unitary operators $u$ which verify that $u-1$ belongs to the $p$-Schatten ideal $\mathcal{B}_{p}(\mathcal{H})$. Let $\mathcal{O}$ be a smooth manifold on which $U_{p}(\mathcal{H})$ acts transitively and smoothly. Then one can endow $\mathcal{O}$ with a natural Finsler metric in terms of the $p$-Schatten norm and the action of $U_{p}(\mathcal{H})$. Our main result establishes that for any pair of given initial conditions

$$
x \in \mathcal{O} \text { and } X \in(T \mathcal{O})_{x}
$$

there exists a curve $\delta(t)=e^{t z} \cdot x$ in $\mathcal{O}$, with $z$ a skew-hermitian element in the $p$-Schatten class, such that

$$
\delta(0)=x \text { and } \dot{\delta}(0)=X,
$$

which remains minimal as long as $t\|z\|_{p} \leq \pi / 4$. Moreover, $\delta$ is unique with these properties. We also show that the metric space $(\mathcal{O}, d)$ (where $d$ is the rectifiable distance) is complete. In the process we establish minimality results in the groups $U_{p}(\mathcal{H})$ and a convexity property for the rectifiable distance. As an example of these spaces, we treat the case of the unitary orbit

$$
\mathcal{O}=\left\{u A u^{*}: u \in U_{p}(\mathcal{H})\right\}
$$

of a self-adjoint operator $A \in \mathcal{B}(\mathcal{H})$.
\end{abstract}

\section{INTRODUCTION}

Let $\mathcal{H}$ be an infinite dimensional Hilbert space and $\mathcal{B}(\mathcal{H})$ the space of bounded linear operators acting in $\mathcal{H}$. Denote by $\mathcal{B}_{p}(\mathcal{H})$ the $p$-Schatten class

$$
\mathcal{B}_{p}(\mathcal{H})=\left\{a \in \mathcal{B}(\mathcal{H}): \operatorname{Tr}\left(\left(a^{*} a\right)^{p / 2}\right)<\infty\right\},
$$

where $\operatorname{Tr}$ is the usual trace of $\mathcal{B}(\mathcal{H})$. In this paper we shall focus on the case when $p$ is an even integer. The spaces $\mathcal{B}_{p}(\mathcal{H})$ are Banach spaces with the norms

$$
\|a\|_{p}=\operatorname{Tr}\left(\left(a^{*} a\right)^{p / 2}\right)^{1 / p} .
$$

We use the subscript $h$ (resp. $a h$ ) to denote the sets of hermitian (resp. skewhermitian) operators, e.g. $\mathcal{B}_{p}(\mathcal{H})_{h}=\left\{x \in \mathcal{B}_{p}(\mathcal{H}): x^{*}=-x\right\}$. Throughout this paper, \|\| denotes the usual operator norm. Denote by $G l(\mathcal{H})$ the linear group

Received by the editors December 19, 2007.

2000 Mathematics Subject Classification. Primary 22E65; Secondary 58B20, 58E50.

This work was partially supported by IAM-CONICET.

(C)2009 American Mathematical Society Reverts to public domain 28 years from publication 
and by $U(\mathcal{H})$ the unitary group of $\mathcal{H}$. Consider the following classical Banach-Lie groups of operators [12]:

$$
G l_{p}(\mathcal{H})=\left\{g \in G l: g-1 \in \mathcal{B}_{p}(\mathcal{H})\right\}
$$

and

$$
U_{p}(\mathcal{H})=\left\{u \in U(\mathcal{H}): u-1 \in \mathcal{B}_{p}(\mathcal{H})\right\},
$$

where $1 \in \mathcal{B}(\mathcal{H})$ denotes the identity operator. These groups have differentiable structure when endowed with the metric $\left\|g_{1}-g_{2}\right\|_{p}$ (note that $g_{1}-g_{2} \in \mathcal{B}_{p}(\mathcal{H})$ ). For instance, the Banach-Lie algebra of $U_{p}(\mathcal{H})$ is the (real) Banach space $\mathcal{B}_{p}(\mathcal{H})_{a h}$.

Let $\mathcal{O}$ be a topological space on which $U_{p}(\mathcal{H})$ acts transitively, such that for any element $x \in \mathcal{O}$, the subgroup $G_{x}=\left\{u \in U_{p}(\mathcal{H}): u \cdot x=x\right\}$ is a closed submanifold of $U_{p}(\mathcal{H})$. This implies that $\mathcal{O}$ can be endowed with a differentiable manifold structure in a way such that the map

$$
\pi=\pi_{x}: U_{p}(\mathcal{H}) \rightarrow \mathcal{O}, \quad \pi_{x}(u)=u \cdot x
$$

is a smooth submersion. In other words, $\mathcal{O} \simeq U_{p}(\mathcal{H}) / G_{x}$ is a smooth homogeneous space of the group $U_{p}(\mathcal{H})$. The main object of this paper is the geometric study of this space, under reasonably general conditions, which are specified below. We introduce a Finsler metric $\left\{\|\|_{x}: x \in \mathcal{O}\right\}$ in $\mathcal{O}$ (a Riemannian metric if $p=2$ ) induced by the $p$ norm in $\mathcal{B}_{p}(\mathcal{H})$ and by the action. We focus on the existence of metric geodesics, i.e. curves of minimal length. Our approach is to study the metric geometry of the group $U_{p}(\mathcal{H})$ in order to obtain results in $\mathcal{O}$. In the process we find properties in $U_{p}(\mathcal{H})$ which we claim are interesting in their own right. For instance:

(1) The one-parameter unitary groups $e^{t z} \in U_{p}(\mathcal{H})\left(z \in \mathcal{B}_{p}(\mathcal{H})_{a h}\right)$, regarded as curves of unitaries, have minimal length in the $p$-norm, as long as $t\|z\| \leq \pi$ (note that this condition is given in terms of the usual norm $\|z\|$ of $z$, a fact that implies that there are arbitrarily long minimal curves in $U_{p}(\mathcal{H})$ ).

(2) The map $f_{p}(t)=d_{p}\left(u_{0}, e^{t z}\right)^{p}$, where $d_{p}$ is the rectifiable metric induced by the $p$-norm and $u_{0}$ is a fixed element in $U_{p}(\mathcal{H})$, is a strictly convex function, provided that $u_{0}$ and the endpoints of the curve lie at a distance not greater than $\pi / 4$.

Denote by $\mathcal{G}_{x}$ the Banach-Lie algebra of $G_{x}$. We shall make the assumption that $G_{x}$ is locally exponential: since any element $u \in U_{p}(\mathcal{H})$ is of the form $u=e^{z}$ for some $z \in \mathcal{B}_{p}(\mathcal{H})$, we ask that for any element $v \in G_{x}$ close to $1 \in G_{x}$ there exists an element $z \in \mathcal{G}_{x}$ such that $v=e^{z}$. Apparently, if this holds for a given $x_{0} \in \mathcal{O}$, then it holds for any $x \in \mathcal{O}$ (since the groups $G_{x}$ and $G_{x_{0}}$ are conjugate by an inner automorphism). This is equivalent to asking that $G_{x}$ is a (non complemented) Banach-Lie subgroup of $U_{p}(\mathcal{H})$.

Using these facts we prove our main results on $\mathcal{O}$ :

(1) If $x \in \mathcal{O}$ and $X \in(T \mathcal{O})_{x}$, then there exists a unique curve $\gamma(t)=e^{t z} \cdot x$ with $\gamma(0)=x$ and $\dot{\gamma}(0)=X$, which has minimal length in $\mathcal{O}$ as long as $t\|X\|_{x} \leq \pi / 4$.

(2) The metric space $(\mathcal{O}, d)$ is complete, where $d$ is the rectifiable metric induced by the Finsler metric in $\mathcal{O}$.

There are many examples of this situation. For instance, if $A \in \mathcal{B}(\mathcal{H})$ is a selfadjoint operator, its unitary orbit $\mathcal{O}_{A}=\left\{u A u^{*}: u \in U_{p}(\mathcal{H})\right\}$ is a homogeneous space, and the group $G_{A}$ consists of the elements of $U_{p}(\mathcal{H})$ which commute with 
A. $G_{A}$ is a Banach-Lie subgroup of $U_{p}(\mathcal{H})$ since it is an algebraic subgroup (cf. Theorem 4.13 in [5]), and its Lie algebra is given by

$$
\mathcal{G}_{A}=\left\{x \in \mathcal{B}_{p}(\mathcal{H})_{a h}: x A-A x=0\right\} .
$$

Unitary orbits of operators have been previously studied from a geometric point of view in [3, 4, 6, 7, 10, 16, 19. In this particular framework, restricting the action to these classical groups $U_{p}(\mathcal{H})$, certain results can be found in [6, 7, 8, 16.

Let us briefly describe the contents of the paper. In Section 2 we introduce the Finsler metric which is Riemannian if $p=2$. In Section 3 we examine the metric structure of the group $U_{p}(\mathcal{H})$ endowed with the Finsler metric given by the $p$-norm. We recall certain known facts and prove results which we believe are new, among them the two results described above. In Section 4 we show the consequence of these facts on the homogeneous space $\mathcal{O}$ : existence and uniqueness of short curves with given initial data. In Section 5 we prove that the metric spaces $\mathcal{O}$ are complete. Section 6 is devoted to the example $\mathcal{O}_{A}, p=2$, giving a characterization of the case when $\mathcal{O}_{A}$ is a smooth submanifold of the affine Hilbert space $A+\mathcal{B}_{2}(\mathcal{H})_{h}$. In Section 7 we state what we believe is the main open problem in this setting, namely the existence of minimal curves joining given endpoints in $\mathcal{O}$, and prove a partial positive result.

\section{LINEAR CONNECTIONS AND METRICS}

Let us first consider the case $p=2$. One can induce a metric in (the tangent spaces of) $\mathcal{O}$ by means of the decomposition

$$
\mathcal{B}_{2}(\mathcal{H})_{a h}=\mathcal{G}_{x} \oplus \mathcal{F}_{x}
$$

where $\mathcal{F}_{x}$ is the $\operatorname{Tr}$-orthogonal complement of $\mathcal{G}_{x}$. Apparently, $\mathcal{F}_{x}$ is invariant by the inner action of $G_{x}$. Therefore this decomposition defines what in classical geometry of homogeneous spaces [22] is called a Reductive Structure.

The kernel of $d\left(\pi_{x}\right)_{1}$ is $\mathcal{G}_{x}$, therefore

$$
\delta_{x}:=\left.d\left(\pi_{x}\right)_{1}\right|_{\mathcal{F}_{x}}: \mathcal{F}_{x} \rightarrow(T \mathcal{O})_{x}
$$

is a linear isomorphism. Denote by $\kappa_{x}$ its inverse, and by $P_{x}$ the $T r$-orthogonal projection

$$
P_{x}: \mathcal{B}_{2}(\mathcal{H})_{a h} \rightarrow \mathcal{F}_{x} \subset \mathcal{B}_{2}(\mathcal{H})_{a h} .
$$

We endow $(T \mathcal{O})_{x}$ with the following inner product:

$$
\langle V, W\rangle_{x}=\operatorname{Tr}\left(\kappa_{x}(W)^{*} \kappa_{x}(V)\right)=-\operatorname{Tr}\left(\kappa_{x}(W) \kappa_{x}(V)\right), \quad V, W \in(T \mathcal{O})_{x} .
$$

Clearly the distribution $x \mapsto\langle,\rangle_{x}$ is smooth, in the sense that that if $V, W$ are tangent fields in $\mathcal{O}$, then the map $\mathcal{O} \ni x \mapsto\left\langle V_{x}, W_{x}\right\rangle_{x}$ is smooth, and therefore (2.1) defines a Riemann-Hilbert metric in $\mathcal{O}$.

The Levi-Civita connection of this metric can be computed. In the paper [17] two natural linear connections for a homogeneous reductive space were introduced. The first, which is called the reductive connection $\nabla^{r}$, is analogous to the connection that one obtains for a reductive manifold in finite dimensions. It can be described as follows. If $V$ is a tangent field and $W$ is a tangent vector (at $x$ ) in $\mathcal{O}$, then

$$
\kappa_{x}\left(\nabla_{W}^{r} V(x)\right)=\kappa_{x}(W)\left(\kappa_{x}\left(V_{x}\right)\right)+\left[\kappa_{x}\left(V_{x}\right), \kappa_{x}(W)\right],
$$

where [, ] is the commutator of operators in $\mathcal{B}(\mathcal{H})$ and $a(b)$ denotes the derivative of $b$ in the direction of $a$. 
A straightforward computation shows that since the maps $\kappa$ are isometric, the reductive connection is compatible with the metric defined.

The second natural connection for a reductive space is the classifying connection $\nabla^{c}$. Suppose that $V, W$ are as above; then put

$$
\nabla_{V}^{c}(W)(x)=\delta_{x} P_{x}\left(\kappa_{x}(V)\left[\kappa_{x}(W)\right]_{x}\right) .
$$

These two connections share the same geodesics, which are described below, and have torsion tensors with opposite signs. It follows that the connection

$$
\nabla=\frac{1}{2}\left(\nabla^{r}+\nabla^{c}\right)
$$

has zero torsion and the same geodesics. We claim that this connection $\nabla$ is the Levi-Civita connection of the metric (2.1) introduced above, in the sense that it is symmetric (torsion free) and compatible with the metric. To prove this claim, it only remains to show that $\nabla^{c}$ is compatible with the metric.

Lemma 2.1. The classifying connection $\nabla^{c}$ is compatible with the metric $\langle,\rangle_{x}$ in $\mathcal{O}$.

Proof. Let $V(t), W(t)$ be two tangent fields along the curve $\nu(t)$ in $\mathcal{O}$. Then

$$
\left\langle\frac{D^{c} V}{d t}, W\right\rangle_{\nu}=-\operatorname{Tr}\left(\kappa_{\nu}(W) \kappa_{\nu}\left(\frac{D^{c} V}{d t}\right)\right)=-\operatorname{Tr}\left(\kappa_{\nu}(W) P_{\nu}\left(\kappa_{\nu}(V)\right)\right)
$$

Note that since $\kappa_{\nu}(W) \in R\left(P_{\nu}\right), \operatorname{Tr}\left(\kappa_{\nu}(W) P_{\nu}\left(\kappa_{\nu}(V)\right)\right)=\operatorname{Tr}\left(\kappa_{\nu}(W) \kappa_{\nu}(V)\right)$. Analogously

$$
\left\langle V, \frac{D^{c} W}{d t}\right\rangle_{\nu}=-\operatorname{Tr}\left(\kappa_{\nu}(W) \kappa_{\nu}(V)\right)
$$

Then

$$
\begin{aligned}
\left\langle\frac{D^{c} V}{d t}, W\right\rangle_{\nu}+\left\langle V, \frac{D^{c} W}{d t}\right\rangle_{\nu} & =-\operatorname{Tr}\left(\kappa_{\nu}(W) \kappa_{\nu}(V)\right)-\operatorname{Tr}\left(\kappa_{\nu}(W) \kappa_{\nu}(V)\right) \\
& =\frac{d}{d t}\langle V, W\rangle_{\nu}
\end{aligned}
$$

The geodesics of these connections are computed explicitly in [17. For instance, the geodesic $\gamma$ with $\gamma(0)=x$ and $\dot{\gamma}(0)=V$ is given by

$$
\gamma(t)=e^{\kappa_{x}(V)} \cdot x, t \in \mathbb{R}
$$

In other words, geodesics of $\mathcal{O}$ are of the form $e^{t z} \cdot x$, for $z \in \mathcal{F}_{x}$.

The following linear differential equation is usually called the horizontal lifting equation of the reductive structure:

$$
\left\{\begin{array}{l}
\dot{\Gamma}=\kappa_{\gamma}(\dot{\gamma}) \Gamma, \\
\Gamma(0)=1
\end{array}\right.
$$

It is a linear differential equation in $\mathcal{B}_{2}(\mathcal{H})$. In order to assure the existence and uniqueness of solutions, one must check that the mapping

$$
[0,1] \ni t \mapsto \kappa_{\gamma(t)}(\dot{\gamma}(t)) \in \mathcal{B}_{2}(\mathcal{H})_{a h}
$$

is smooth. This is clear if $\gamma$ is smooth.

Therefore the equation (2.5) has, for a given $\gamma$, a unique solution. One can prove, as in classical homogeneous reductive spaces [22], the following result. 
Proposition 2.2. Let $\gamma(t), t \in[0,1]$, be a smooth curve in $\mathcal{O}$. Then the unique solution $\Gamma$ of (2.5) verifies

(1) $\Gamma(t) \in U_{2}(\mathcal{H}), t \in[0,1]$.

(2) $\Gamma$ lifts $\gamma: \pi_{\gamma}(\Gamma)=\gamma$.

(3) $\Gamma$ is horizontal: $\Gamma^{*} \dot{\Gamma} \in \mathcal{F}_{\gamma}$.

It will be useful to take a brief look at the natural Riemannian geometry of the group $U_{2}(\mathcal{H})$. Namely, the metric given by considering the trace inner product and, therefore, the 2-norm at each tangent space. The tangent spaces of $U_{2}(\mathcal{H})$ are

$$
\left(T U_{2}(\mathcal{H})\right)_{u}=u \mathcal{B}_{2}(\mathcal{H})_{a h}=\mathcal{B}_{2}(\mathcal{H})_{a h} u
$$

The covariant derivative consists of differentiating in the ambient space and projecting (orthogonally with respect to the real part of the trace) onto $T U_{2}(\mathcal{H})$. Geodesics of the Levi-Civita connection are curves of the form

$$
\mu(t)=u e^{t x}
$$

for $u \in U_{2}(\mathcal{H})$ and $x \in \mathcal{B}_{2}(\mathcal{H})_{a h}$. The exponential mapping of this connection is the map

$$
\exp : \mathcal{B}_{2}(\mathcal{H})_{a h} \rightarrow U_{2}(\mathcal{H}), \quad \exp (x)=e^{x} .
$$

In the general case $p>2$, one can endow the homogeneous space $\mathcal{O}$ with a Finsler metric, derived from the $p$-norm and the group action. Following ideas in [11, we shall not consider a linear connection in this case, but focus only on characterizing short curves (or metric geodesics) which are not the geodesics of any linear connection. First let us introduce some notation. The action of $U_{p}(\mathcal{H})$ on $\mathcal{O}$ induces two kinds of maps. If one fixes $x \in \mathcal{O}$, one has the submersion

$$
\pi_{x}: U_{p}(\mathcal{H}) \rightarrow \mathcal{O}, \quad \pi_{x}(u)=u \cdot x, \quad u \in U_{p}(\mathcal{H}) .
$$

If one fixes $u \in U_{p}(\mathcal{H})$, one has the diffeomorphism

$$
\ell_{u}: \mathcal{O} \rightarrow \mathcal{O}, \quad \ell_{u}(x)=u \cdot x, \quad x \in \mathcal{O} .
$$

If $x \in \mathcal{O}$ and $X \in(T \mathcal{O})_{x}$, put

$$
\|X\|_{x}=\inf \left\{\|z\|_{p}: z \in \mathcal{B}_{p}(\mathcal{H})_{a h},\left(d \pi_{x}\right)_{1}(z)=X\right\} .
$$

This metric could be called the quotient metric of $\mathcal{O}$, because it is the quotient metric in the Banach space $(T \mathcal{O})_{x}$ if one identifies it with $\mathcal{B}_{p}(\mathcal{H})_{a h} / \mathcal{G}_{x}$. Indeed, since $\mathcal{G}_{x}=\operatorname{ker}\left(d \pi_{x}\right)_{1}$, if $z \in \mathcal{B}_{p}(\mathcal{H})_{a h}$ with $\left(d \pi_{x}\right)_{1}(z)=X$, then

$$
\|X\|_{x}=\inf \left\{\|z-y\|_{p}: y \in \mathcal{G}_{x}\right\} .
$$

Note that if $p=2$, this metric coincides with the previously defined Riemannian metric. Indeed, if $Q_{x}=1-P_{x}$ is the orthogonal projection onto $\mathcal{G}_{x}$, then each $z \in \mathcal{B}_{2}(\mathcal{H})_{a h}$ can be uniquely decomposed as

$$
z=z-Q_{x}(z)+Q_{x}(z)=z_{0}+Q_{x}(z) .
$$

Hence

$$
\|z-y\|_{2}^{2}=\left\|z_{0}+Q_{x}(z)-y\right\|_{2}^{2}=\left\|z_{0}\right\|_{2}^{2}+\left\|Q_{x}(z)-y\right\|_{2}^{2} \geq\left\|z_{0}\right\|_{2}^{2}
$$

for any $y \in \mathcal{G}_{2, x}$, which shows that

$$
\|X\|_{x}=\inf \left\{\|z-y\|_{2}: y \in \mathcal{G}_{x}\right\}=\left\|z_{0}\right\|_{2},
$$

where $z_{0}$ is the unique vector in $\mathcal{G}_{x}^{\perp}$ such that $\left(d \pi_{x}\right)_{1}\left(z_{0}\right)=X$. 
This metric in $\mathcal{O}$ is invariant by the group action (i.e. the group acts isometrically on the tangent spaces): if $x \in \mathcal{O}, X \in(T \mathcal{O})_{x}$ and $u \in U_{p}(\mathcal{H})$,

$$
\left\|\left(d \ell_{u}\right)_{x}(X)\right\|_{u \cdot x}=\|X\|_{x} .
$$

Indeed, if $z \in \mathcal{B}_{p}(\mathcal{H})_{a h}$, then $\left(d \pi_{u \cdot x}\right)_{1}(z)=\left(d \ell_{u}\right)_{x}(X)$ if and only if

$$
\begin{aligned}
X & =\left(\left(d \ell_{u}\right)_{x}\right)^{-1} \circ\left(d \pi_{u \cdot x}\right)_{1}(z)=\left(d \ell_{u^{-1}}\right)_{u \cdot x} \circ\left(d \pi_{u \cdot x}\right)_{1}(z) \\
& =\left(d \ell_{u^{-1}} \circ \pi_{u \cdot x}\right)_{1}(z)=\left(d \pi_{x}\right)_{1}(z) .
\end{aligned}
$$

Throughout this paper, $L$ denotes the length functional for piecewise smooth curves in $\mathcal{O}$, measured with the quotient norm just defined,

$$
L(\gamma)=\int_{t_{0}}^{t_{1}}\|\dot{\gamma}(t)\|_{\gamma} d t
$$

and $d$ denotes the rectifiable distance in $\mathcal{O}$,

$$
d\left(x_{0}, x_{1}\right)=\inf \left\{L(\gamma): \gamma \subset \mathcal{O} \text { joins } x_{0} \text { and } x_{1}\right\} .
$$

\section{Metric structure of $U_{p}(\mathcal{H})$}

In this section we recall and complete certain facts from 1 concerning the minimality of geodesics in $U_{p}(\mathcal{H})$. Afterwards we establish local convexity results for the geodesic distance. These results will be the key in obtaining minimality results in $\mathcal{O}$. Proofs for these statements for the case $p=2$ can be found in 1 .

Throughout, $L_{p}$ denotes the length functional for piecewise smooth curves in $U_{p}(\mathcal{H})$, measured with the $p$-norm:

$$
L_{p}(\alpha)=\int_{t_{0}}^{t_{1}}\|\dot{\alpha}(t)\|_{p} d t
$$

and $d_{p}$ is the rectifiable distance in $U_{p}(\mathcal{H})$ :

$$
d_{p}\left(u_{1}, u_{2}\right)=\inf \left\{L_{p}(\gamma): \gamma \subset U_{p}(\mathcal{H}) \text { joins } u_{1} \text { and } u_{2}\right\} .
$$

Remark 3.1. (1) The exponential map

$$
\exp : \mathcal{B}_{p}(\mathcal{H})_{a h} \rightarrow U_{p}(\mathcal{H})
$$

is surjective.

(2) The exponential map is a bijection between the sets

$$
\mathcal{B}_{p}(\mathcal{H})_{a h} \supset\left\{z \in \mathcal{B}_{p}(\mathcal{H})_{a h}:\|z\|<\pi\right\} \rightarrow\left\{u \in U_{p}(\mathcal{H}):\|1-u\|<2\right\} .
$$

(3) Moreover,

$$
\exp :\left\{z \in \mathcal{B}_{p}(\mathcal{H})_{a h}:\|z\| \leq \pi\right\} \rightarrow U_{p}(\mathcal{H})
$$

is surjective.

These facts can be obtained from the following observation. If $u \in U_{p}(\mathcal{H})$, then it has a spectral decomposition $u=p_{0}+\sum_{k \geq 1}\left(1+\alpha_{k}\right) p_{k}$, where $\alpha_{k}$ are the nonzero eigenvalues of $u-1 \in \mathcal{B}_{p}(\mathcal{H})$. There exist $t_{k} \in \mathbb{R}$ with $\left|t_{k}\right| \leq \pi$ such that $e^{i t_{k}}=1+\alpha_{k}$. The elementary estimate

$$
\left|t_{k}\right|^{p}\left(1-\frac{\left|t_{k}\right|^{2}}{12}\right)^{p / 2} \leq\left|e^{i t_{k}}-1\right|^{p}=\left|\alpha_{k}\right|^{p}
$$

implies that the element $z=\sum_{k \geq 1} i t_{k} p_{k}$, whose exponential is $u$, lies in $\mathcal{B}_{p}(\mathcal{H})_{a h}$. 
The following result states that the one parameter groups of unitaries in $U_{p}(\mathcal{H})$ have minimal length up to a certain critical value of $t$. This could be derived from the general theory of Hilbert-Riemann manifolds for the case $p=2$. In any case, the proof, which is essentially contained in 11, is operator theoretic and provides a uniform lower bound for the geodesic radius.

Theorem 3.2. The following facts hold:

(1) Let $u \in U_{p}(\mathcal{H})$ and $x \in \mathcal{B}_{p}(\mathcal{H})_{\text {ah }}$ with $\|x\| \leq \pi$. Then the curve $\mu(t)=u e^{t x}$, $t \in[0,1]$, is shorter than any other piecewise smooth curve in $U_{p}(\mathcal{H})$ joining the same endpoints. Moreover if $\|x\|<\pi, \mu$ is unique with this property.

(2) Let $u_{0}, u_{1} \in U_{p}(\mathcal{H})$. Then there exists a minimal geodesic curve joining them. If $\left\|u_{0}-u_{1}\right\|<2$, this geodesic is unique.

(3) There are in $U_{p}(\mathcal{H})$ minimal geodesics of arbitrary length. Thus the diameter of $U_{p}(\mathcal{H})$ is infinite.

(4) If $u, v \in U_{p}(\mathcal{H})$, then

$$
\sqrt{1-\frac{\pi^{2}}{12}} d_{p}(u, v) \leq\|u-v\|_{p} \leq d_{p}(u, v) .
$$

In particular the metric space $\left(U_{p}(\mathcal{H}), d_{p}\right)$ is complete.

Proof. Concerning the first statement, in [1] the following was proved. If $u \in U_{2}(\mathcal{H})$ and $x \in \mathcal{B}_{2}(\mathcal{H})_{a h}$ with $\|x\| \leq \pi$, then the curve $\mu(t)=u e^{t x}$ is minimal for $t \in[0,1]$, when the length is measured with the $p$-norm. Clearly it suffices to treat the case $u=1$. Suppose that there exists a curve $\gamma(t) \in U_{p}(\mathcal{H})$ with $L_{p}(\gamma)<L_{p}(\mu)+\epsilon$. One can approximate $x$ with a skew-hermitian operator $z$ of finite spectrum with the following properties:

(1) $\|z\| \leq\|x\| \leq \pi$.

(2) $\|x\|_{p}-\epsilon / 2<\|z\|_{p} \leq\|x\|_{p}$.

(3) There exists a $C^{\infty}$ curve of unitaries joining $e^{x}$ and $e^{z}$, of $p$-length less than $\epsilon / 2$.

The first two conditions are clear. The third can be obtained as follows. Put $e^{-x} e^{z}=e^{y}$, with $y \in \mathcal{B}_{p}(\mathcal{H})_{a h}$. The element $z$ can be adjusted so as to obtain $y$ of arbitrarily small $p$-norm. Then the curve of unitaries $\nu(t)=e^{x} e^{t y}$ is $C^{\infty}$, and joins $e^{x}$ and $e^{z}$ with $p$-length $\|y\|_{p}<\epsilon / 2$.

Now consider the curve $\gamma_{1}$, which is the curve $\gamma$ followed by the curve $e^{x} e^{t y}$ above. Then clearly

$$
L_{p}\left(\gamma_{1}\right) \leq L_{p}(\gamma)+\|y\|_{p}<L_{p}(\gamma)+\epsilon / 2 .
$$

Note that $L_{p}\left(\gamma_{1}\right)<\|x\|_{p}-\epsilon / 2$ and that $\gamma_{1}$ joins 1 and $e^{z}$. We claim that there exists a curve $\gamma_{2}$ in $U_{2}(\mathcal{H})$, also joining 1 and $e^{z}$, with length $L_{p}\left(\gamma_{2}\right)<L_{p}\left(\gamma_{1}\right)+\epsilon / 4$. Indeed, the curve $\gamma_{1}$ is of the form $\gamma_{1}(t)=e^{\alpha(t)}$ for a continuous piecewise $C^{1}$ path $\alpha \in \mathcal{B}_{p}(\mathcal{H})_{a h}$ with endpoints 0 and $z$. By compactness of the unit interval, one can uniformly approximate $\alpha$ by a curve $\beta$ with the same endpoints, lying in $\mathcal{B}_{2}(\mathcal{H})_{a h}$, in order for $\gamma_{2}(t)=e^{\beta(t)}$ to verify our claim. These facts imply that the curve $\gamma_{2}$ in $U_{2}(\mathcal{H})$ which joins 1 and $e^{z}$ is shorter than the curve $e^{t z}$ (which lies in $U_{2}(\mathcal{H}$ ) because the spectrum of $z$ is finite). This contradicts the minimality statement in $U_{2}(\mathcal{H})$ proved in [1].

Let us prove that if $\|x\|<\pi$, then $\mu$ is unique with the minimality property. To do this we shall follow a standard procedure, using the first variation formula for 
the functional $F_{p}$ which is given by

$$
F_{p}(\gamma)=\int_{0}^{1}\|\dot{\gamma}(t)\|_{p}^{p} d t
$$

if $\gamma(t) \in U_{p}(\mathcal{H}), t \in[0,1]$.

Let $\gamma_{s}(t), t \in[0,1], s \in(-r, r)$, be a smooth variation of the curve $\gamma$, i.e.

(1) $\gamma_{s}(t) \in U_{p}(\mathcal{H})$, for all $s, t$.

(2) The map $(s, t) \mapsto \gamma_{s}(t)$ is smooth.

(3) $\gamma_{0}(t)=\gamma(t)$.

We shall use a formula for

$$
\left.\frac{d}{d s} F_{p}\left(\gamma_{s}\right)\right|_{s=0}
$$

obtained in 2] in the context of a $C^{*}$-algebra with trace, which applies here because the formal computations are the same (they only involve partial derivatives and integration by parts). As in classical differential geometry, we shall call the expression obtained the first variation formula. Let

$$
V_{s}=\frac{d}{d t} \gamma_{s} \quad \text { and } \quad W_{s}=\frac{d}{d s} \gamma_{s}
$$

With lower case types we denote the left translations

$$
v_{s}=\gamma_{s}^{*} V_{s} \text { and } w_{s}=\gamma_{s}^{*} W_{s} .
$$

Note that $V_{s}, W_{s} \in\left(T U_{p}(\mathcal{H})\right)_{\gamma_{s}}$, whereas $v_{s}, w_{s} \in \mathcal{B}_{p}(\mathcal{H})_{a h}$.

Then

$$
\frac{(-1)^{p / 2}}{p} \frac{d}{d s} F_{p}\left(\gamma_{s}\right)=\left.\operatorname{Tr}\left(v_{s}^{p-1} w_{s}\right)\right|_{t=0} ^{t=1}-\int_{0}^{1} \operatorname{Tr}\left(\frac{d}{d t}\left[v_{s}^{p-1}\right] w_{s}\right) d t .
$$

Suppose that $\gamma(t) \in U_{p}(\mathcal{H})$ is a smooth minimal curve, and let $\gamma_{s}(t)$ be a variation with fixed endpoints $\gamma(0)$ and $\gamma(1)$, i.e. $\gamma_{s}(0)=\gamma(0)$ and $\gamma_{s}(1)=\gamma(1)$ for all $s$. Then $\left.\frac{d}{d s} F_{p}\left(\gamma_{s}\right)\right|_{s=0}=0$, and thus

$$
0=\left.\operatorname{Tr}\left(v_{0}^{p-1} w_{0}\right)\right|_{t=0} ^{t=1}-\int_{0}^{1} \operatorname{Tr}\left(w_{0} \frac{d}{d t}\left(v_{0}^{p-1}\right)\right) d t .
$$

The fixed endpoints hypothesis implies that the first term vanishes. Then

$$
\int_{0}^{1} \operatorname{Tr}\left(w_{0} \frac{d}{d t}\left(v_{0}^{p-1}\right)\right) d=0
$$

for any variation $\gamma_{s}$ with fixed endpoints. Let us denote this field by $Z(t)=\frac{d}{d t}\left(v_{0}^{p-1}\right)$ and by $A(t)=w_{0}(t)$. Both $A$ and $Z$ are continuous fields, $A$ in $\mathcal{B}_{p}(\mathcal{H})_{a h}$ and $Z$ in $\mathcal{B}_{q}(\mathcal{H})_{a h}$, where $1 / p+1 / q=1$. The variation formula implies that

$$
\int_{0}^{1} \operatorname{Tr}(A(t) Z(t)) d t=0
$$

for any continuous field $A$ in $\mathcal{B}_{p}(\mathcal{H})_{a h}$ such that $A(0)=A(1)=0$. We claim that this condition implies that $Z(t)=0$ for all $t$.

First note that the requirement that the field $A$ vanishes at 0 and 1 can be removed: let $f_{r}(t)$ be a real function which is constant and equal to 1 in the interval $[r, 1-r]$ and such that $f(0)=f(1)=0$, with $0 \leq f_{r}(t) \leq 1$ for all $t$. Let $B(t)$ be any continuous field in $\mathcal{B}_{p}(\mathcal{H})_{a h}$ and consider $A_{r}(t)=f_{r}(t) B(t)$. Then $\int_{0}^{1} A_{r}(t) Z(t) d t=0$, and if $r \rightarrow 0, \int_{0}^{1} B(t) Z(t) d t=0$. Also it is clear that the 
integral will vanish if $A$ is nonskew-hermitian. Indeed, this is clear if $A$ is hermitian, and for general $A$, decompose $A$ as the sum of its hermitian and skew-hermitian parts.

Fix $t_{0}$ in the interval $[0,1]$. Let $Z\left(t_{0}\right)=u\left|Z\left(t_{0}\right)\right|$ be the polar decomposition and consider $x=\left|Z\left(t_{0}\right)\right|^{q-1} u^{*} \in \mathcal{B}_{p}(\mathcal{H})$. Consider the constant field $A(t)=x$ in $\mathcal{B}_{p}(\mathcal{H})$; then

$$
0=\int_{0}^{1} \operatorname{Tr}(x Z(t)) d t=\left\|Z\left(t_{0}\right)\right\|_{q}^{q}
$$

Then $v_{0}^{p-1}$ is constant, and since $p$ is even and $v_{0}$ is skew-hermitian, $v_{0}(t)=$ $\gamma(t)^{*} \frac{d}{d t} \gamma(t)$ is constant, i.e. $\gamma(t)=e^{t x}$ for some $x \in \mathcal{B}_{p}(\mathcal{H})_{a h}$.

Fact (2) was proved in [1] the (algebraic) argument for $p>2$ is the same as for $p=2$.

Fact (3) was proved in [1].

Fact (4) follows from the elementary estimate in the remark above.

Let us establish further facts on the metric structure of the group $U_{p}(\mathcal{H})$.

Lemma 3.3. Let $a, b \in \mathcal{B}_{p}(\mathcal{H})$, let $\exp : \mathcal{B}_{p}(\mathcal{H}) \rightarrow 1+\mathcal{B}_{p}(\mathcal{H})$ be $\exp (x)=e^{x}$, and let ad $a: \mathcal{B}_{p}(\mathcal{H}) \rightarrow \mathcal{B}_{p}(\mathcal{H})$ be the operator ad $a(x)=x a-$ ax. Then

$$
d \exp _{a}(b)=\int_{0}^{1} e^{(1-t) a} b e^{t a} d t=e^{a} F(a d a) b=F(a d a)\left(e^{a} b\right),
$$

where $F(z)=\frac{e^{z}-1}{z}=\sum_{n \geq 0} \frac{z^{n}}{(n+1) !}$. The differential is invertible at a if and only if $\sigma(\operatorname{ad} a) \cap\{2 k \pi i\}=\emptyset\left(k \in \mathbb{Z}_{\neq 0}\right)$, and then

$$
d \exp _{a}^{-1}(w)=e^{-a} F(a d a)^{-1} w .
$$

In particular, if $\|a\|<\pi$, then $d \exp _{a}$ is invertible. If $a \in \mathcal{B}_{p}(\mathcal{H})_{a h}$, then the differential is a contraction:

$$
\left\|d \exp _{a}(b)\right\|_{p} \leq\|b\|_{p}
$$

Proof. Compute $\lim _{s \rightarrow 0} \frac{e^{a+s b}-e^{a}}{s}$, applied to the identity

$$
e^{a+b}-e^{a}=\int_{0}^{1} e^{(1-t) a} b e^{t(a+b)}
$$

which is elementary and can be proven integrating by parts the functions $f(t)=$ $e^{(1-t) a}$ and $g(t)=e^{t(a+b)}$ in $[0,1]$. To prove the second equality, write

$$
e^{-t a} b e^{t a}=e^{t\left(R_{a}-L_{a}\right)}(b),
$$

where $L_{a}(x)=a x$ and $R_{a}(x)=x a$ denote left and right multiplication by $a$. Then $\int_{0}^{1} e^{t\left(R_{a}-L_{a}\right)} d t=\sum_{n \geq 0} \frac{1}{n !} \int_{0}^{1} t^{n} d t\left(R_{a}-L_{a}\right)^{n}=\sum_{n \geq 0} \frac{1}{(n+1) !}\left(R_{a}-L_{a}\right)^{n}=F(\operatorname{ad} a)$. If $\|a\|<\pi$, then $\|\operatorname{ad} a\|<2 \pi$ and hence $\sigma(\operatorname{ad} a) \subset B(0,2 \pi)$, so the spectrum of ad $a$ does not intersect the zero set of $F$. The last assertion is due to the fact that, when $a$ is skew-hermitian, $e^{a}$ is a unitary element; hence

$$
\left\|d \exp _{a}(b)\right\|_{p} \leq \int_{0}^{1}\left\|e^{(1-t) a} b e^{t a}\right\|_{p} d t=\|b\|_{p} .
$$


The following elementary lemma will simplify the proof of the next theorem.

Lemma 3.4. Let $C, \varepsilon>0$, and let $f(-\varepsilon, 1+\varepsilon) \rightarrow \mathbb{R}$ be a nonconstant real analytic function such that $f^{\prime}(s)^{2} \leq C f^{\prime \prime}(s)$ for any $s \in[0,1]$. Then $f$ is strictly convex in $(0,1)$.

Proof. By the mean value theorem, the condition on $f$ implies that for each pair of roots of $f^{\prime}$, there is another root of $f^{\prime}$ in between. Since $f$ is analytic and nonconstant, the set of roots of $f^{\prime}$ is an empty set or has one point $\alpha \in(-\varepsilon, 1+\varepsilon)$. If this set of roots does not intersect $(0,1)$, then $f^{\prime \prime}>0$ there and we are done. We assume then that there exists $\alpha$ in $(0,1)$ such that $f^{\prime}(\alpha)=0$. Note that $-f^{\prime}(x)=f^{\prime}(\alpha)-f^{\prime}(x)=\int_{x}^{\alpha} f^{\prime \prime}(s) d s>0$ for any $x \in(-\varepsilon, \alpha]$ and $f^{\prime}(y)=f^{\prime}(y)-$ $f_{p}^{\prime}(\alpha)=\int_{\alpha}^{y} f^{\prime \prime}(s) d s>0$ for any $y \in[\alpha, 1+\varepsilon)$. Hence $f^{\prime}$ is strictly negative in $(-\varepsilon, \alpha)$ and strictly positive in $(\alpha, 1+\varepsilon)$, so $f$ is strictly convex in each interval. If $f(\alpha)<[f(1)-f(0)] \alpha+f(0)$, we are done. If not, by the mean value theorem there exists $x \in(0, \alpha), y \in(\alpha, 1)$ such that

$$
f(1)-f(0)=\frac{f(\alpha)-f(0)}{\alpha}=f^{\prime}(x)<0
$$

and

$$
f(1)-f(0)=\frac{f(1)-f(\alpha)}{1-\alpha}=f^{\prime}(y)>0,
$$

a contradiction.

Remark 3.5. The Hessian of the $p$-norms was studied in [2, 18]. We recall a few facts we will use in the proof of the next theorem. Let $a, b, c \in \mathcal{B}_{p}(\mathcal{H})_{a h}$, and let $H_{a}: \mathcal{B}_{p}(\mathcal{H})_{a h} \rightarrow \mathbb{R}$ stand for the symmetric bilinear form given by

$$
H_{a}(b, c)=(-1)^{\frac{p}{2}} p \sum_{k=0}^{p-2} \operatorname{Tr}\left(a^{p-2-k} b a^{k} c\right) .
$$

If $Q$ is the quadratic form associated to $H$, then (cf. Lemma 4.1 in [2] and equation (3.1) in [18):

(1) $Q_{a}([b, a]) \leq 4\|a\|_{\infty}^{2} Q_{a}(b)$.

(2) $Q_{a}(b)=p\left\|b a^{\frac{p}{2}-1}\right\|_{2}^{2}+\frac{p}{2} \sum_{l+m=n-2}\left\|a^{l}(a b+b a) a^{m}\right\|_{2}^{2}$.

In particular $H_{a}$ is positive definite for any $a \in \mathcal{B}_{p}(\mathcal{H})_{a h}$.

Our convexity results follow. If $u \in U_{p}(\mathcal{H})$, denote by $B_{p}(u, r)$ the geodesic ball of radius $r$ around $u$ in $U_{p}(\mathcal{H})$, i.e.

$$
B_{p}(u, r)=\left\{w \in U_{p}(\mathcal{H}): d_{p}(u, w)<r\right\} .
$$

Theorem 3.6. Let $p$ be a positive even integer, $u \in U_{p}(\mathcal{H})$ and $\beta:[0,1] \rightarrow U_{p}(\mathcal{H})$ be a non-constant geodesic contained such that $\beta \subset B_{p}\left(u, \frac{\pi}{2}\right)$. Assume further that $u$ does not belong to any prologantion of $\beta$. Then

$$
f_{p}(s)=d_{p}(u, \beta(s))^{p}
$$

is a strictly convex function.

Proof. We may assume that $u=1$ since the action of unitary elements is isometric. Let $v, z \in \mathcal{B}_{p}(\mathcal{H})_{a h}$ such that $\beta(s)=e^{v} e^{s z}$. Since $\beta \subset B_{p}\left(1, \frac{\pi}{2}\right)$, it has an analytic $\operatorname{logarithm} w_{s}=\log (\beta(s))=\log \left(e^{v} e^{s z}\right)$. Let $\gamma_{s}(t)=e^{t w_{s}}$. Since $\left\|w_{s}\right\| \leq\left\|w_{s}\right\|_{p}<$ 
$\pi / 2, \gamma_{s}$ is a short geodesic joining 1 and $\beta(s)$, of length $\left\|w_{s}\right\|_{p}=d_{p}(1, \beta(s))$. Then $f_{p}(s)=\left\|w_{s}\right\|_{p}^{p}=\operatorname{Tr}\left(\left(-w_{s}^{2}\right)^{\frac{p}{2}}\right)=(-1)^{\frac{p}{2}} \operatorname{Tr}\left(w_{s}^{p}\right)$, hence

$$
f_{p}^{\prime}(s)=(-1)^{\frac{p}{2}} p \operatorname{Tr}\left(w_{s}^{p-1} \dot{w}_{s}\right)=\frac{1}{p-1} H_{w_{s}}\left(\dot{w}_{s}, w_{s}\right) .
$$

For $x, y \in \mathcal{B}_{a h}^{p}$, we have the formula $d \exp _{x}(y)=\int_{0}^{1} e^{(1-t) x} y e^{t x} d t$ from the previous lemma. Since $e^{w_{s}}=e^{v} e^{s z}$, then $e^{-w_{s}} d \exp _{w_{s}}\left(\dot{w}_{s}\right)=z$, namely

$$
z=\int_{0}^{1} e^{-t w_{s}} \dot{w}_{s} e^{t w_{s}} d t
$$

Thus $\operatorname{Tr}\left(w_{s}^{p-1} \dot{w}_{s}\right)=\int_{0}^{1} \operatorname{Tr}\left(w_{s}^{p-1} e^{-t w_{s}} \dot{w}_{s} e^{t w_{s}}\right) d t=\operatorname{Tr}\left(z w_{s}^{p-1}\right)$. Hence

$$
f_{p}^{\prime \prime}(s)=(-1)^{\frac{p}{2}} p \sum_{k=0}^{p-2} \operatorname{Tr}\left(w_{s}^{p-2-k} \dot{w}_{s} w_{s}^{k} z\right)=H_{w_{s}}\left(\dot{w}_{s}, z\right),
$$

and again by equation (3.1) above, if we put $\delta_{s}(t)=e^{-t w_{s}} \dot{w}_{s} e^{t w_{s}}$, then

$$
f_{p}^{\prime \prime}(s)=\int_{0}^{1} H_{w_{s}}\left(\delta_{s}(0), \delta_{s}(t)\right) d t .
$$

Suppose that for this value of $s \in[0,1], R_{s}^{2}:=Q_{w_{s}}\left(\dot{w}_{s}\right) \neq 0$, where $Q_{w_{s}}$ is the quadratic form associated to $H_{w_{s}}$. If $K_{s} \subset \mathcal{B}_{p}(\mathcal{H})_{a h}$ is the null space of $H_{w_{s}}$, consider the quotient space $\mathcal{B}_{p}(\mathcal{H})_{a h} / K_{s}$ equipped with the inner product $H_{w_{s}}(\cdot, \cdot)$. An elementary computation shows that $\delta_{s}(t)$ lives in a sphere of radius $R_{s}$ of this pre-Hilbert space. Hence

$$
H_{w}\left(\delta_{s}(0), \delta_{s}(t)\right)=R_{s}^{2} \cos \left(\alpha_{s}(t)\right),
$$

where $\alpha_{s}(t)$ is the angle subtended by $\delta_{s}(0)$ and $\delta_{s}(t)$. Then, reasoning in the sphere

$$
\begin{aligned}
R_{s} \alpha_{s}(t) & \leq L_{0}^{t}\left(\delta_{s}\right)=\int_{0}^{t} Q_{w_{s}}^{\frac{1}{2}}\left(e^{-t w_{s}}\left[w_{s}, \dot{w}_{s}\right] e^{t w_{s}}\right) d t \\
& =\int_{0}^{t} Q_{w_{s}}^{\frac{1}{2}}\left(\left[w_{s}, \dot{w}_{s}\right]\right) d t=t Q_{w_{s}}^{\frac{1}{2}}\left(\left[w_{s}, \dot{w}_{s}\right]\right) .
\end{aligned}
$$

By property (1) of the above remark,

$$
R_{s} \alpha_{s}(t) \leq t 2\left\|w_{s}\right\|_{\infty} R_{s} \leq 2 t\left\|w_{s}\right\|_{p} R_{s}<R_{s} \pi
$$

if $\left\|w_{s}\right\|_{p}<\frac{\pi}{2}$. So

$$
\cos \left(\alpha_{s}(t)\right) \geq \cos \left(2 t\left\|w_{s}\right\|_{p}\right)
$$

and then integrating with respect to the $t$-variable,

$$
f_{p}^{\prime \prime}(s) \geq R_{s}^{2} \frac{\sin \left(2\left\|w_{s}\right\|_{p}\right)}{2\left\|w_{s}\right\|_{p}}>0
$$

provided $R_{s} \neq 0$. On the other hand, the Cauchy-Schwarz inequality for $H_{w_{s}}$ shows that if $R_{s}=0$, then

$$
(p-1) f_{p}^{\prime}(s)=H_{w_{s}}\left(w_{s}, \dot{w}_{s}\right) \leq Q_{w_{s}}^{\frac{1}{2}}\left(\dot{w}_{s}\right) Q_{w_{s}}^{\frac{1}{2}}\left(w_{s}\right)=0 .
$$

Assume that $R_{s}$ is identically zero, $s \in[0,1]$. Then $f_{p}$ is constant with $f_{p}(s)=$ $f_{p}(0)=\|v\|_{p}$ for any $s \in[0,1]$. Moreover, by property (2) of the above remark, $R_{s}=0$ implies $w_{s}^{\frac{p}{2}-1} z=0$ and an elementary computation involving the functional 
calculus of skew-adjoint operators shows that $w_{s} z=0$. In particular $v z=0$, which implies $w_{s}=v+s z$ by the Baker-Campbell-Hausdorff formula. But since the norm of $\mathcal{B}_{p}(\mathcal{H})$ is strictly convex, $w_{s}$ cannot have constant norm unless $v$ is a multiple of $z$, and in that case, $u$ and $\beta$ are aligned, contradicting the assumption of the theorem. So there is at least one point $s_{0} \in[0,1]$ where $R_{s_{0}} \neq 0$, so $f_{p}$ is nonconstant and by Lemma 3.4, $f_{p}$ is strictly convex since

$$
(p-1)^{2} f_{p}^{\prime}(s)^{2}=H_{w_{s}}^{2}\left(w_{s}, \dot{w}_{s}\right) \leq Q_{w_{s}}\left(\dot{w}_{s}\right) Q_{w_{s}}\left(w_{s}\right) \leq C f_{p}^{\prime \prime}(s) .
$$

Remark 3.7. A careful reading of the proof of the above theorem shows that $f_{p}$ is in fact strictly convex provided that the uniform norm $\left\|w_{s}\right\|$ is strictly less than $\pi / 2$.

Corollary 3.8. Let $u_{1}, u_{2}, u_{3} \in U_{p}(\mathcal{H})$ with $u_{2}, u_{3} \in B_{p}\left(u_{1}, \frac{\pi}{4}\right)$, and assume that they are not aligned (i.e. they do not lie in the same geodesic). Let $\gamma(s)$ be the short geodesic joining $u_{2}$ with $u_{3}$. Then dist ${ }_{p}\left(u_{1}, \gamma(s)\right)<\frac{\pi}{2}$ for $s \in[0,1]$ and $\frac{\pi}{4}$ is the radius of convexity of the metric balls of $U_{p}(\mathcal{H})$.

Proof. Note that

$$
\begin{aligned}
\operatorname{dist}_{p}\left(u_{1}, \gamma(s)\right) & \leq \operatorname{dist}_{p}\left(u_{1}, u_{2}\right)+\frac{1}{2} \operatorname{dist}_{p}\left(u_{2}, u_{3}\right) \\
& \leq \operatorname{dist}_{p}\left(u_{1}, u_{2}\right)+\frac{1}{2}\left(\operatorname{dist}_{p}\left(u_{2}, u_{1}\right)+\operatorname{dist}_{p}\left(u_{3}, u_{1}\right)\right)<2 \frac{\pi}{4}=\frac{\pi}{2}
\end{aligned}
$$

hence the conclusion follows from the previous theorem.

\section{Minimality in $\mathcal{O}$ : Initial values problem}

For our main result on minimality in $\mathcal{O}$, we make the assumption that for some (hence for any) $x \in \mathcal{O}$, the group $G_{x}$ is locally exponential. Namely, there exists a radius $\delta>0$ such that if $v \in G_{x}$ with $\|v-1\|_{p}<\delta$, then there exists an element $z \in$ $\mathcal{G}_{x}$ such that $v=e^{z}$. This is equivalent to the fact that $G_{x}$ is a (noncomplemented) Banach-Lie subgroup of $U_{p}(\mathcal{H})$. This property implies in particular, that $G_{x}$ is locally geodesically convex: given any pair of elements $v_{1}, v_{2} \in G_{x}$ with $\left\|v_{1}-v_{2}\right\|_{p}<$ $\delta$, then there exists a unique minimal geodesic of $U_{p}(\mathcal{H})$, which lies inside $G_{x}$, and joins $v_{1}$ and $v_{2}$.

Our argument on minimality in $\mathcal{O}$ will consist of comparing the lengths of the liftings of curves to the unitary group $U_{p}(\mathcal{H})$. For the case $p=2$ this technique is based on the following fact:

Remark 4.1. Let $\gamma(t), t \in[0,1]$, be a smooth curve in $\mathcal{O}$, with $\gamma(0)=x$, and let $\Gamma$ be its horizontal lifting. Then

$$
L_{2}(\Gamma)=L(\gamma)
$$

Indeed, recall from (2.5) that $\dot{\Gamma}=\kappa_{\gamma}(\dot{\gamma}) \Gamma$, and also note that by definition of the metric, $\kappa_{x}:(T \mathcal{O})_{x} \rightarrow \mathcal{F}_{x} \subset \mathcal{B}_{2}(\mathcal{H})_{a h}$ is isometric. Then

$$
\|\dot{\gamma}\|_{\gamma}=\left\|\kappa_{\gamma}(\dot{\gamma})\right\|_{2}=\left\|\Gamma^{*} \dot{\Gamma}\right\|_{2}=\|\dot{\Gamma}\|_{2}
$$

and the result follows. 
Let us show that for $p>2$ we can still have isometric lifts of curves in $\mathcal{O}$.

First note that the general theory ensures the existence of piecewise $C^{1}$ liftings in $U_{p}(\mathcal{H})$ of $C^{1}$ curves in $\mathcal{O}$, due to the fact that for any fixed $x \in \mathcal{O}$, the map

$$
\pi_{x}: U_{p}(\mathcal{H}) \rightarrow \mathcal{O}, \pi_{x}(u)=u \cdot x
$$

is a submersion.

First we need to discuss the projection to closed linear spaces in $\mathcal{B}_{p}(\mathcal{H})$ and a few technical lemmas.

Remark 4.2. Let $1<p<\infty$. Then for any convex closed set $S \subset \mathcal{B}_{p}(\mathcal{H})_{a h}$ there exists a continuous map $Q_{S}: \mathcal{B}_{p}(\mathcal{H})_{a h} \rightarrow S$ which sends $x \in \mathcal{B}_{p}(\mathcal{H})_{a h}$ to its best approximant $Q_{S}(x) \in S$, i.e.

$$
\left\|x-Q_{S}(x)\right\|_{p} \leq\|x-s\|_{p}
$$

for any $s \in S$.

The map $Q_{S}$ is single-valued and continuous, because $\mathcal{B}_{p}(\mathcal{H})$ is uniformly convex and uniformly smooth (see for instance [9]). Note that

$$
\|Q(x)\|_{p} \leq\|Q(x)-x\|_{p}+\|x\|_{p} \leq\|0-x\|_{p}+\|x\|_{p}=2\|x\|_{p}
$$

and also that

$$
\left\|x-Q_{S}(x)-s\right\|_{p} \geq\left\|x-Q_{S}(x)\right\|_{p}
$$

for any $s \in S$; hence $Q_{S}\left(x-Q_{S}(x)\right)=0$, namely $Q_{S} \circ\left(1-Q_{S}\right)=0$. Also, for any positive $\lambda \in \mathbb{R}$,

$$
Q_{S}(\lambda x)=\lambda Q_{S}(x) .
$$

Let $x \in \mathcal{O}$, let $G=G_{x}$ be the isotropy group and let $\mathcal{G}_{x}$ be the Lie algebra of $G$ as usual. Let $Q=Q_{\mathcal{G}_{x}}$ be the projection to the best approximant in $\mathcal{G}_{x}$. Let

$$
\mathcal{G}_{x}^{\perp_{p}}=Q^{-1}(0)=\left\{x \in \mathcal{B}_{p}(\mathcal{H})_{a h}:\|x\|_{p} \leq\|x-y\|_{p} \text { for any } y \in \mathcal{G}_{x}\right\} .
$$

Then any element $z \in \mathcal{B}_{p}(\mathcal{H})_{a h}$ can be decomposed as

$$
z=z-Q(z)+Q(z)
$$

where $z-Q(z) \in \mathcal{G}_{x}^{\perp_{p}}$ and $Q(z) \in \mathcal{G}_{x}$. In particular, these facts imply that given $x \in \mathcal{O}$ and $X \in(T \mathcal{O})_{x}$, there exists a minimal lifting $z_{0} \in \mathcal{B}_{p}(\mathcal{H})_{a h}$ for $x$. Indeed, since

$$
\pi_{x}: U_{p}(\mathcal{H}) \rightarrow \mathcal{O}, \pi_{x}(u)=u \cdot x
$$

is a smooth submersion, the differential $\left(d \pi_{x}\right)_{1}$ is surjective, and thus there exists $z \in \mathcal{B}_{p}(\mathcal{H})_{a h}$ such that $d\left(\pi_{x}\right)_{1}(z)=X$. Then a minimal lifting is

$$
z_{0}=z-Q(z) \in \mathcal{G}_{x}^{\perp_{p}} .
$$

Calling $\bar{Q}=1-Q$, we have

$$
\mathcal{G}_{x}=\bar{Q}^{-1}(0)=\operatorname{Im}(Q), \quad \mathcal{G}_{x}^{\perp_{p}}=Q^{-1}(0)=\operatorname{Im}(\bar{Q}),
$$

and also

$$
\bar{Q}^{2}=\bar{Q}, \quad Q^{2}=Q, \quad \bar{Q} \circ Q=Q \circ \bar{Q}=0 .
$$

Lemma 4.3. Let $p$ be an even positive integer. Let $x \in \mathcal{O}$ and $X \in(T \mathcal{O})_{x}$. An element $z \in \mathcal{B}_{p}(\mathcal{H})_{a h}$ with $\left(d \pi_{x}\right)_{1}(z)=X$ is a minimal lifting for $X$ if and only if $\operatorname{Tr}\left(z^{p-1} y\right)=0$ for all $y \in \mathcal{G}_{x}$. For any $X \in(T \mathcal{O})_{x}$ there exists a unique minimal lifting $z \in \mathcal{G}_{x}^{\perp_{p}}$ such that $\|z\|_{p}=\|X\|_{x}$. 
Proof. Suppose that $z_{0}$ is a minimal lifting, and for a fixed $y \in \mathcal{G}_{x}$, let $f(t)=$ $\left\|z_{0}-t y\right\|_{p}^{p}$. Then $f$ is a smooth map with a minimum at $t=0$, i.e. $f^{\prime}(0)=0$. A straightforward computation shows that $f^{\prime}(t)=\operatorname{Tr}\left(\left(z_{0}-t y\right)^{p-1} y\right)$, and thus $\operatorname{Tr}\left(z_{0}^{p-1} y\right)=0$. Conversely, suppose that $\operatorname{Tr}\left(z_{0}^{p-1} y\right)=0$ for all $y \in \mathcal{G}_{x}$ and suppose that there exists $y_{0} \in \mathcal{G}_{x}$ such that $\left\|z_{0}-y_{0}\right\|_{p}<\left\|z_{0}\right\|_{p}$. Then the map $f(t)=\left\|z_{0}-t y_{0}\right\|_{p}^{p}$ would not have a minimum at $t=0$. This is a contradiction, since $f$ is convex and $f^{\prime}(0)=0$. The existence of minimal liftings was established in the previous remark: take any $w \in \mathcal{B}_{p}(\mathcal{H})_{a h}$ such that $\left(d \pi_{x}\right)_{1}(w)=X$ and then take $z=w-Q_{\mathcal{G}_{x}}(w)$. If $\left(d \pi_{x}\right)_{1}\left(z_{1}\right)=\left(d \pi_{x}\right)_{1}\left(z_{2}\right)=X$ for $z_{1}, z_{2} \in \mathcal{B}_{p}(\mathcal{H})_{a h}$, then $z_{1}-z_{2} \in \mathcal{G}_{x}$. If $z_{1}$ and $z_{2}$ are minimal liftings of $X$, then we have

$$
\left\|z_{1}\right\|_{p} \leq\left\|z_{1}-\left(z_{1}-z_{2}\right)\right\|_{p}=\left\|z_{2}\right\|_{p}
$$

and the reversed inequality also holds, hence $\left\|z_{1}\right\|_{p}=\left\|z_{2}\right\|_{p}=\|X\|_{x}$. To prove uniqueness we may then assume that $\left\|z_{1}\right\|_{p}=\left\|z_{2}\right\|_{p}=1$. Consider the smooth convex function $g: \mathcal{G}_{x} \rightarrow \mathbb{R}_{>0}$ given by

$$
y \mapsto\left\|z_{1}-y\right\|_{p}^{p} .
$$

Now $g(0)=\left\|z_{1}\right\|_{p}^{p}=1$ is a minimum for $g$, and we are assuming that $g\left(z_{1}-z_{2}\right)=$ $\left\|z_{2}\right\|_{p}^{p}=1$ is another minimum. Hence $g$ must be constant on the straight segment $s\left(z_{1}-z_{2}\right) \in \mathcal{G}_{x}$ for any $s \in[0,1]$. In particular (with $s=\frac{1}{2}$ ),

$$
\left\|\frac{1}{2}\left(z_{1}+z_{2}\right)\right\|_{p}^{p}=\left\|z_{1}\right\|_{p}^{p}=\left\|z_{2}\right\|_{p}^{p}=1
$$

which forces $z_{1}=z_{2}$, since $\mathcal{B}_{p}(\mathcal{H})$ is uniformly convex.

Having established the linear result on minimal liftings, let us prove two technical lemmas in order to extend the isometric lifting property to smooth curves $\gamma \subset \mathcal{O}$.

Lemma 4.4. Let $k \geq 1, w \in \mathcal{B}_{p}(\mathcal{H})$ with $\|w\|_{p}<\frac{\pi}{2}$. Then

is invertible in $\mathcal{B}\left(\mathcal{B}_{p}(\mathcal{H})\right)$ and

$$
T=1+\frac{(a d w)^{2}}{4 k^{2} \pi^{2}}
$$

$$
\left\|T^{-1}\right\| \leq\left(1-\frac{\|w\|^{2}}{k^{2} \pi^{2}}\right)^{-1} \leq\left(1-\frac{\|w\|_{p}^{2}}{k^{2} \pi^{2}}\right)^{-1} .
$$

Proof. Since $\|\operatorname{ad} w\| \leq 2\|w\| \leq 2\|w\|_{p}<\pi$, the map $T$ is invertible and its inverse can be computed with the Neumann series.

Remark 4.5. Consider $g(r)=\frac{r}{\sin (r)}$ with $g(0)=1$. Then $g:[0, \pi) \rightarrow \mathbb{R}$ is positive and increasing, and from the Weierstrass expansion of $\sin (z)$ we obtain

$$
g(z)=\prod_{k \geq 1}\left(1-\frac{z^{2}}{k^{2} \pi^{2}}\right)^{-1}
$$

for any $z$ such that $|z|<\pi$.

Proposition 4.6. Let $F(z)=\frac{e^{z}-1}{z}, g(r)=\frac{r}{\sin (r)}$, let $w \in \mathcal{B}_{p}(\mathcal{H})$ with $\|w\|_{p}<$ $\frac{\pi}{2}$, and let $t \in[0,1]$. Then

$$
\left\|F(a d w)^{-1}\right\| \leq g(\|w\|) \leq g\left(\|w\|_{p}\right) .
$$


Proof. The Weierstrass expansion of $F(z)=\frac{e^{z}-1}{z}$ is given by

$$
F(z)=\prod_{k \geq 1}\left(1+\frac{z^{2}}{4 k^{2} \pi^{2}}\right)
$$

where the product converges uniformly on compact sets to $F$. Then $F(\operatorname{ad} w)$ is invertible since $\|$ ad $w \|<\pi$ and

$$
F(\operatorname{ad} w)^{-1}=\prod_{k \geq 1}\left(1+\frac{(\operatorname{ad}(w))^{2}}{4 k^{2} \pi^{2}}\right)^{-1} .
$$

Hence

$$
\left\|F(\operatorname{ad} w)^{-1}\right\| \leq \prod_{k \geq 1}\left(1-\frac{\|w\|^{2}}{k^{2} \pi^{2}}\right)^{-1}=g(\|w\|) \leq g\left(\|w\|_{p}\right)
$$

by the previous lemma.

Lemma 4.7. Let $1<p<\infty, x \in \mathcal{O}$ and let $Q=Q_{\mathcal{G}_{x}}$ be the best approximant projection. Let $\Gamma \subset U_{p}(\mathcal{H})$ be a piecewise $C^{1}$ curve parametrized in the interval $[0,1]$. Then there exists a piecewise $C^{1}$ curve $z:[0,1] \rightarrow \mathcal{G}_{x}$ with $z(0)=0$ such that

$$
F(a d z) \dot{z}=-Q\left(\Gamma^{*} \dot{\Gamma}\right)
$$

If $u_{\Gamma}=e^{z} \in G_{x}$, then $u_{\Gamma}:[0,1] \rightarrow \mathcal{B}_{p}(\mathcal{H})$ obeys the differential equation

$$
u_{\Gamma} u_{\Gamma}^{*}=-Q\left(\Gamma^{*} \dot{\Gamma}\right)
$$

and $L_{p}\left(u_{\Gamma}\right) \leq 2 L_{p}(\Gamma)$.

Proof. Assume first that $\Gamma$ is $C^{1}$ in the whole $[0,1]$. Let $R_{0}=\max _{t \in \bar{J}}\|\dot{\Gamma}\|_{p}$, where $J$ is an open interval containing $[0,1]$ where $\Gamma$ is differentiable. Let $0<R<\frac{\pi}{2}$ and put $\mathcal{G}=\mathcal{G}_{x}$. Then if $x \in \mathcal{G} \cap B(0, R)$, the map $F(\operatorname{ad} x)$ is invertible by the previous lemma, its inverse is analytic and can be written as a power series in ad $x$, and hence

$$
F(\operatorname{ad} x)^{-1}: \mathcal{G} \rightarrow \mathcal{G}
$$

because $\mathcal{G}$ is a Banach-Lie algebra. Moreover, since $g$ is increasing,

$$
\left\|F(\operatorname{ad} x)^{-1}\right\| \leq g\left(\|x\|_{p}\right) \leq g(R) .
$$

Let $f: J \times B(0, R) \cap \mathcal{G} \rightarrow \mathcal{G}$ be given by

$$
f(t, x)=-F(\operatorname{ad} x)^{-1} Q_{\mathcal{G}}\left(\Gamma^{*}(t) \dot{\Gamma}(t)\right) .
$$

Then $f$ is continuous since $Q$ and $F^{-1}$ are continuous. Moreover,

$$
\|f(t, x)\|_{p} \leq\left\|F(\operatorname{ad} x)^{-1}\right\| 2\|\dot{\Gamma}(t)\|_{p} \leq g(R) 2 R_{0}=L
$$

by Remark 4.2 and the previous lemma. Since $H(\operatorname{ad} x)=F(\operatorname{ad} x)^{-1}$ is analytic in the ball $\|x\|_{p}<\frac{\pi}{2}$, we have

$$
\|H(\operatorname{ad} x)-H(\operatorname{ad} y)\| \leq C(R)\|\operatorname{ad} x-\operatorname{ad} y\| \leq 2 C(R)\|x-y\|_{p},
$$

where $C(R)$ is the bound for $H^{\prime}$ in $\|z\|_{p} \leq R$. Then

$$
\|f(t, x)-f(t, y)\|_{p} \leq 4 C(R) R_{0}\|x-y\|_{p}=K\|x-y\|_{p} .
$$


Then $f$ satisfies a Lipschitz condition, uniformly respect to $t \in J$. Hence by Proposition 1.1 of $\mathrm{Ch}$. IV in [15], there exists a continuous solution $z_{0}:(-b, b) \times$ $B(0, R / 4) \rightarrow \mathcal{G} \cap B(0, R)$ of the integral equation

$$
z(t)=\int_{0}^{t} f(s, z(s)) d s
$$

with $z_{0}(0)=0$. Here $b$ is any real number

$$
0<b<\frac{R}{4 L K}=\frac{\sin (R)}{32 C(R) R_{0}^{2}} .
$$

Note that $z_{0}$ is in fact $C^{1}$. Differentiating both sides and multiplying by $F(\operatorname{ad} z(t))$ gives the equation stated. We have proved so far that the equation

$$
F(\operatorname{ad} z) \dot{z}=-Q\left(\Gamma^{*} \dot{\Gamma}\right)
$$

has a local solution defined around zero. By a standard argument, it follows that one can find a piecewise $C^{1}$ solution defined on the whole interval $[0,1]$ : let $N \in \mathbb{N}$ such that $\frac{1}{N}<b$, and let $t_{k}=\frac{k}{N}$. Then $\left[t_{k}, t_{k+1}\right](k=0,1, \ldots, N)$ is a partition of $[0,1]$ such that the integral equation

$$
z(t)=\int_{t_{k}}^{t_{k+1}} f(s, z(s)) d s
$$

with the initial conditions $z_{0}(0)=0, z_{k}\left(t_{k}\right)=z_{k-1}\left(t_{k}\right)$ for $k \geq 1$, has a solution $z_{k}:\left[t_{k}, t_{k+1}\right] \rightarrow \mathcal{G}$. Then the curve $z_{1} \sharp z_{2} \sharp \cdots \sharp z_{N}$ is a piecewise $C^{1}$ solution of the equation defined in the whole $[0,1]$.

If $\Gamma$ is piecewise $C^{1}$ instead of $C^{1}$, one might replace the argument above for a similar argument in each of the intervals where $\Gamma$ is $C^{1}$, and use the continuity of $\Gamma$ to state the boundary conditions for $z$.

If $u_{\Gamma}(t)=e^{z(t)}$, then

$$
\dot{u}_{\Gamma}(t)=d \exp _{z(t)}(\dot{z}(t))=\int_{0}^{1} e^{s z(t)} \dot{z}(t) e^{-s z(t)} d s u_{\Gamma}(t)=F(\operatorname{ad} z(t)) \dot{z}(t) u_{\Gamma}(t)
$$

by Lemma 3.3. Then $\dot{u}=F(\operatorname{ad} z) \dot{z} u$, and hence $\dot{u} u^{*}=-Q\left(\Gamma^{*} \dot{\Gamma}\right)$. Thus

$$
\|\dot{u}\|_{p}=\left\|Q\left(\Gamma^{*} \dot{\Gamma}\right)\right\|_{p} \leq 2\left\|\Gamma^{*} \dot{\Gamma}\right\|_{p}=2\|\dot{\Gamma}\|_{p},
$$

and therefore $L_{p}(u) \leq 2 L_{p}(\Gamma)$.

Proposition 4.8. Let $x_{0} \in \mathcal{O}$, and let $\gamma=\Gamma \cdot x_{0} \subset \mathcal{O}$ be a $C^{1}$ curve defined in an interval containing $[0,1]$. Then $\gamma$ admits a piecewise $C^{1}$ lift $\beta \subset U_{p}(\mathcal{H})$ (that is, $\left.\beta \cdot x_{0}=\gamma\right)$ such that $L(\gamma)=L_{p}(\beta) \leq L_{p}(\Gamma)$. We shall call $\beta$ an isometric lift of $\gamma$.

Proof. Let $u=u_{\Gamma}=e^{z}$ be the curve of the previous lemma. Then $\beta=\Gamma u_{\gamma}$ is a lift of $\gamma$ because $u \in G$. Moreover,

$$
\begin{aligned}
\|\dot{\beta}\|_{p} & =\|\dot{\Gamma} u+\Gamma \dot{u}\|_{p}=\left\|\Gamma^{*} \dot{\Gamma}+\dot{u} u^{*}\right\|_{p}=\left\|\Gamma^{*} \dot{\Gamma}-Q\left(\Gamma^{*} \dot{\Gamma}\right)\right\|_{p} \\
& =\min _{y \in \mathcal{G}}\left\|\Gamma^{*} \dot{\Gamma}-y\right\|_{p} \leq\left\|\Gamma^{*} \dot{\Gamma}\right\|_{p}=\|\dot{\Gamma}\|_{p},
\end{aligned}
$$

hence $L(\gamma)=L_{p}(\beta) \leq L_{p}(\Gamma)$.

In order to simplify the exposition, we assume that the subgroup $G_{x}$ is exponential. 
Theorem 4.9. Let $p$ be a positive even integer, $x \in \mathcal{O}, X \in(T \mathcal{O})_{x}$, and let $z_{0} \in \mathcal{B}_{p}(\mathcal{H})_{a h}$ be a minimal lifting for $X$. Then the curve

$$
\delta(t)=e^{t z_{0}} \cdot x,
$$

which verifies $\delta(0)=x$ and $\dot{\delta}(0)=X$, has minimal length $\left\|z_{0}\right\|_{p}$ in the interval $[0,1]$ if $\left\|z_{0}\right\|_{p}<\pi / 4$. Moreover, the curve $\delta$ is unique with this property, in the sense that if $\gamma \subset \mathcal{O}$ is another curve joining $x$ to $e^{z_{0}} \cdot x$ of length $\left\|z_{0}\right\|_{p}$, then $\gamma(t)=e^{t z_{0}} \cdot x$.

Proof. Let $\gamma$ be a smooth curve in $\mathcal{O}$ with $\gamma(0)=x$ and $\gamma(1)=e^{z_{0}} \cdot x$. Denote by $\beta$ as above an isometric lift of $\gamma$. Note that the curve $\epsilon(t)=e^{t z_{0}}$ is an isometric lift for $\delta$. Then it suffices to compare $\beta$ and $\epsilon$ (note that both curves start at 1 ). There exists in $U_{p}(\mathcal{H})$ a minimal curve $\alpha(t)=e^{t z}$ with $e^{z}=\beta(1)$, with $L_{p}(\alpha) \leq L_{p}(\beta)$. We claim that $L_{p}(\epsilon) \leq L_{p}(\alpha)$, a fact which ends the proof. If $\|z\|_{p}=L_{p}(\alpha)>\pi / 4$, this fact is clear. Suppose that $\|z\|_{p} \leq \pi / 4$. Let $\nu(t)=e^{z_{0}} e^{t y}$ be the minimal geodesic of $U_{p}(\mathcal{H})$, lying inside $e^{z_{0}} G_{x}$ (i.e. $y \in \mathcal{G}_{x}$ ), connecting $e^{z_{0}}$ to $e^{z}$. Then by Theorem 3.6, the map $f_{p}(s)=d_{p}^{p}(1, \nu(s))$ is convex. We claim that $f_{p}^{\prime}(0)=0$, and thus

$$
L_{p}(\epsilon)^{p}=d_{p}(1, \nu(0))^{p}=f_{p}(0) \leq f_{p}(1)=d_{p}(1, \nu(1))^{p}=L_{p}(\alpha)^{p} .
$$

As in the proof of Theorem [3.6. $f_{p}^{\prime}(0)=(-1)^{p / 2} \operatorname{Tr}\left(z_{0}^{p-1} y\right)$, which vanishes by Lemma 4.3, because $z_{0}$ is a minimal lift. If $L(\gamma)=\left\|z_{0}\right\|_{p}$ (i.e. if $\gamma$ is also short), then

$$
f_{p}(1)=\|z\|_{p}^{p} \leq L_{p}(\beta)^{p}=L(\gamma)^{p}=\left\|z_{0}\right\|_{p}^{p}=f_{p}(0),
$$

and then $z=z_{1}$ because $f_{p}$ is strictly convex. In particular $\beta(1)=e^{z_{0}}$ and $L_{p}(\beta)=L_{p}(\epsilon)=\left\|z_{0}\right\|_{p}$. Since $\left\|z_{0}\right\| \leq\left\|z_{0}\right\|_{p}<\pi / 2$, the curve $\epsilon$ is the unique short geodesic joining 1 to $e^{z_{0}}$ in $U_{p}(\mathcal{H})$, and then $\beta=\epsilon$.

\section{Completeness of the metric spaces $\mathcal{O}$}

We prove that the space $\mathcal{O}$ is a complete metric space with the rectifiable metric. Let us first prove an inequality in $U_{p}(\mathcal{H})$ relating the distance among two geodesics with the distance of the endpoints. Throughout we assume that $p$ is a positive even integer.

Theorem 5.1. Let $g(r)=\frac{r}{\sin (r)}$. Let $u, v, w \in U_{p}(\mathcal{H})$ with $v, w \in B_{p}\left(u, r_{0}\right)$ and $r_{0} \in\left[0, \frac{\pi}{4}\right]$. Let $\gamma$ be the short geodesic joining $v$ to $w$. Let $\alpha$ (resp. $\beta$ ) be the short geodesic joining u to $v$ (resp. u to $w$ ). Let $\gamma_{t}$ be the short geodesic joining $\alpha(t)$ with $\beta(t)$. Then

$$
L_{p}\left(\gamma_{t}\right) \leq \operatorname{tg}\left(r_{0}\right) L_{p}(\gamma) \leq \frac{\pi t}{2 \sqrt{2}} L_{p}(\gamma)
$$

for any $t \in[0,1]$.

Proof. We may suppose $u=1$ without loss of generality. Let $\gamma^{t}=e^{t \log (\gamma)}$. Since $\gamma^{t}(0)=\alpha(t)$ and $\gamma^{t}(1)=\beta(t)$, and $\gamma_{t}$ is a short geodesic joining these same endpoints, one has the inequality

$$
L_{p}\left(\gamma_{t}\right) \leq L_{p}\left(\gamma^{t}\right)
$$

Let us use the dot to denote the derivative with respect to the $s$ variable. Then

$$
\dot{\gamma}^{t}=d \exp _{t l o g(\gamma)}\left(t d \log _{\gamma}(\dot{\gamma})\right)=t d \exp _{t \ln (\gamma)}\left(d \exp _{\gamma}^{-1}(\dot{\gamma})\right) \text {. }
$$


Hence

$$
\left\|\dot{\gamma}^{t}\right\|_{p} \leq t\left\|d \exp _{\gamma}^{-1}(\dot{\gamma})\right\|_{p}
$$

by Lemma 3.3. since the differential of the exponential map is a contraction. By the same lemma,

$$
\left\|\dot{\gamma}^{t}\right\|_{p} \leq t \| F\left(\operatorname{ad}(\log (\gamma))^{-1} \dot{\gamma}\left\|_{p} \leq t g(\|\log (\gamma)\|)\right\| \dot{\gamma} \|_{p}\right.
$$

where the last inequality is due to Proposition 4.6. Now by Corollary 3.8, $\|\log (\gamma)\| \leq$ $\|\log (\gamma)\|_{p}<r_{0}<\frac{\pi}{4}$, and since $g$ is increasing in $[0, \pi)$, the term $g(\|\log (\gamma)\|)$ is bounded by $g\left(r_{0}\right)$, which in turn is bounded by $g\left(\frac{\pi}{4}\right)=\frac{\pi}{2 \sqrt{2}}$. Integrating (5.1) with respect to the variable $s$ in $[0,1]$ gives the inequalities for the $p$-lengths.

Corollary 5.2. Let $u_{1}, u_{2}, u_{3} \in U_{p}(\mathcal{H})$ such that $d_{p}\left(u_{i}, u_{j}\right)<r_{0} \leq \frac{\pi}{4}, u_{2}=u_{1} e^{x}$, $u_{3}=u_{1} e^{y}$. Then

$$
\left|d_{p}\left(u_{1}, u_{2}\right)-d_{p}\left(u_{1}, u_{3}\right)\right| \leq\|x-y\|_{p} \leq g\left(r_{0}\right) d_{p}\left(u_{2}, u_{3}\right) .
$$

Proof. The first inequality is just the reversed triangle inequality, since $\|x\|_{p}=$ $d_{p}\left(u_{1}, u_{2}\right)$ and $\|y\|_{p}=d_{p}\left(u_{1}, u_{3}\right)$. By the invariance of the metric under left action of the unitary group, we may assume that $u_{1}=1$. Then for each $t \in[0,1]$ (in the notation of the previous result)

$$
d_{p}\left(e^{t x}, e^{t y}\right)=\left\|\log \left(e^{t x} e^{-t y}\right)\right\|_{p}=L_{p}\left(\gamma_{t}\right),
$$

which is less than or equal to $g\left(r_{0}\right) t d_{p}\left(u_{2}, u_{3}\right)$ by the same proposition. Then

$$
\left\|\frac{1}{t} \log \left(e^{t x} e^{-t y}\right)\right\|_{p} \leq g\left(r_{0}\right) d_{p}\left(u_{2}, u_{3}\right)
$$

and taking the limit $t \rightarrow 0^{+}$gives the result.

Remark 5.3. Recall Clarkson's inequalities [23] for the $\mathcal{B}_{p}(\mathcal{H})$ spaces, $p \in[2,+\infty)$,

$$
2\|x\|_{p}^{p}+2\|y\|_{p}^{p} \leq\|x-y\|_{p}^{p}+\|x+y\|_{p}^{p},
$$

for any $x, y \in \mathcal{B}_{p}(\mathcal{H})$.

Theorem 5.4 (Weak semi-parallelogram law). Let $\gamma$ be a short geodesic in $U_{p}(\mathcal{H})$ and $u \in U_{p}(\mathcal{H})$ such that $d_{p}(u, \gamma)<r_{0} \leq \frac{\pi}{4}$. Then

$$
\frac{1}{2} g\left(r_{0}\right)\left[d_{p}(u, \gamma(0))^{p}+d_{p}(u, \gamma(1))^{p}\right]-d_{p}(u, \gamma(1 / 2))^{p} \geq \frac{1}{2^{p}} L_{p}(\gamma)^{p}
$$

Proof. We may assume that $\gamma(1 / 2)=1$. Then $\gamma(0)=e^{x}, \gamma(1)=e^{-x}$ and $u=e^{y}$ with $x, y \in \mathcal{B}_{p}(\mathcal{H})_{a h}$. Then, by Clarkson's inequality,

$$
\begin{aligned}
\frac{1}{2^{p}} L_{p}(\gamma)^{p} & =\|x\|_{p}^{p} \leq \frac{1}{2}\left[\|x+y\|_{p}^{p}+\|x-y\|_{p}^{p}\right]-\|y\|_{p}^{p} \\
& =\frac{1}{2}\left[\|x+y\|_{p}^{p}+\|x-y\|_{p}^{p}\right]-d_{p}(u, \gamma(1 / 2))^{p} .
\end{aligned}
$$

Now apply Corollary 5.2

Let us finish this section by proving completeness of the geodesic distance.

Theorem 5.5. The metric space $(\mathcal{O}, d)$ is complete. 
Proof. Let $\left\{b_{n}\right\}_{n \geq 1}$ be a Cauchy sequence in $\mathcal{O}$, and fix $\pi / 4 \geq \varepsilon>0$. Then there exists $n_{0}$ such that $d\left(b_{n}, b_{m}\right)<\varepsilon$ if $n, m \geq n_{0}$. Consider the (submersion) map

$$
\pi=\pi_{b_{n_{0}}}: U_{p}(\mathcal{H}) \rightarrow \mathcal{O}, \pi(u)=u \cdot b_{n_{0}} .
$$

For $n, m \geq n_{0}$, let $\gamma_{n, m}$ be a smooth curve in $\mathcal{O}$ joining $b_{n}$ with $b_{m}$ at (respectively) $t=0$ and $t=1$, such that

$$
d\left(b_{n}, b_{m}\right)^{p} \leq L\left(\gamma_{n, m}\right)^{p}<d\left(b_{n}, b_{m}\right)^{p}+\varepsilon .
$$

Then by Proposition 4.8 the curve $\gamma_{n_{0}, m}$ lifts, via $\pi$, to a curve $\mu_{m}$ of $U_{p}(\mathcal{H})$ with $\mu_{m}(0)=1$,

$$
\pi\left(\mu_{m}(t)\right)=\gamma_{n_{0}, m}(t), \quad t \in[0,1],
$$

such that $L_{p}\left(\mu_{m}\right)=L\left(\gamma_{n_{0}, m}\right)$. Denote it by $u_{m}=\mu_{m}(1)$. Then

$$
\varepsilon+d\left(b_{n_{0}}, b_{m}\right)^{p}>L\left(\gamma_{n_{0}, m}\right)^{p}=L_{p}\left(\mu_{m}\right)^{p} \geq d_{p}\left(1, u_{m}\right)^{p} .
$$

For each $n, m \geq n_{0}$, let $v_{n}, z_{n, m} \in \mathcal{B}_{p}(\mathcal{H})_{a h}$ be such that $\omega_{n, m}(t)=e^{v_{n}} e^{t z_{n, m}}$ is the unique minimal geodesic in $U_{p}(\mathcal{H})$ which joins $u_{n}$ and $u_{m}$ at $t=0$ and $t=1$. Then

$$
d_{p}\left(1, \omega_{n, m}(t)\right)<\pi / 4
$$

if $\varepsilon$ is small enough. Hence by Theorem 5.4 if $n, m \geq n_{0}$, then

$$
\begin{aligned}
2 \varepsilon & >\varepsilon+\frac{1}{2} d\left(b_{n_{0}}, b_{n}\right)^{p}+\frac{1}{2} d\left(b_{n_{0}}, b_{m}\right)^{p} \geq \frac{1}{2} d_{p}\left(1, u_{n}\right)^{p}+\frac{1}{2} d_{p}\left(1, u_{m}\right)^{p} \\
& \geq \frac{1}{g(\pi / 4) 2^{p-1}}\left(d_{p}\left(1, \omega_{n, m}(1 / 2)\right)^{p}+d_{p}\left(u_{n}, u_{m}\right)\right)^{p} \geq \frac{1}{g(\pi / 4) 2^{p-1}} d_{p}\left(u_{n}, u_{m}\right)^{p} .
\end{aligned}
$$

It follows that $\left\{u_{n}\right\}_{n \geq 1}$ is a Cauchy sequence in $U_{p}(\mathcal{H})$, which is complete. Therefore the sequence $b_{n}=\pi\left(u_{n}\right)$ is convergent in $\mathcal{O}$.

\section{Submanifold structure of $\mathcal{O}_{A}$}

In this section we consider the case $\mathcal{O}=\mathcal{O}_{A}=\left\{u A u^{*}: u \in U_{2}(\mathcal{H})\right\}$, for a bounded self-adjoint operator $A$, and we study its local structure as a subset of $\mathcal{B}(\mathcal{H})$. An elementary computation shows that all elements in $\mathcal{O}_{A}$ are of the form $A+k$ with $k \in \mathcal{B}_{2}(\mathcal{H})_{h}$. If $A$ itself lies in $\mathcal{B}_{2}(\mathcal{H})$, then $\mathcal{O}_{A} \subset \mathcal{B}_{2}(\mathcal{H})_{h}$. Otherwise, $\mathcal{O}_{A} \subset A+\mathcal{B}_{2}(\mathcal{H})$, which can be regarded as an affine Hilbert space. In either case, a natural question is whether the manifold $\mathcal{O}_{A}$ is a differentiable submanifold of the ambient Hilbert space. This is the purpose of this section. We show that the orbits $\mathcal{O}_{A}$ are not, in general, differentiable submanifolds of $A+\mathcal{B}_{2}(\mathcal{H})$. We show that $\mathcal{O}_{A} \subset \mathcal{B}_{2}(\mathcal{H})_{h}$ is a differentiable submanifold if and only if the spectrum of $A$ is finite.

The obstruction for $\mathcal{O}_{A}$ to be a submanifold is that its tangent spaces may not be closed in $\mathcal{B}_{2}(\mathcal{H})$. The tangent space of $\mathcal{O}_{A}$ at $A$ (i.e. the derivatives at $A$ of smooth curves in $\mathcal{B}_{2}(\mathcal{H})$, lying inside $\mathcal{O}_{A}$ ) is apparently given by

$$
\left(T \mathcal{O}_{A}\right)_{A}=\left\{x A-A x: x \in \mathcal{B}_{2}(\mathcal{H})_{a h}\right\} .
$$

D. Herrero, D. Voiculescu, C. Apostol and L. Fialkow, among others, established several important results on the closedness of commutators (see the books [4, 14] and the references therein for a complete review on the subject). In particular, L. Fialkow [13] addressed the problem of the spectral characterization of Rosenblum's operators restricted to the Schatten ideals. Let us cite Fialkow's result: denote by $\tau_{A B}$ the operator $\tau_{A B}(x)=A x-x B$. Let $\mathcal{J}$ be any Schatten ideal. 
Theorem 6.1 (Fialkow [13). The following are equivalent:

(1) $\tau_{A B}: \mathcal{B}(\mathcal{H}) \rightarrow \mathcal{B}(\mathcal{H})$ is bounded below.

(2) $\tau_{A B}: \mathcal{J} \rightarrow \mathcal{J}$ is bounded below for some $\mathcal{J}$.

(3) $\tau_{A B}: \mathcal{J} \rightarrow \mathcal{J}$ is bounded below for any $\mathcal{J}$.

(4) $\sigma_{l}(A) \cap \sigma_{r}(B)=\emptyset$.

Here $\sigma_{l}(A)$ (resp. $\left.\sigma_{r}(B)\right)$ denotes the left (resp. right) spectrum of $A$ (resp. $B$ ). Recall the map

$$
\pi_{A}: U_{2}(\mathcal{H}) \rightarrow \mathcal{O}_{A}, \quad \pi_{A}(u)=u A u^{*}
$$

and its differential at the identity

$$
\delta_{A}: \mathcal{B}_{2}(\mathcal{H})_{a h} \rightarrow\left(T \mathcal{O}_{A}\right)_{A}, \quad \delta_{A}(x)=x A-A x .
$$

The Banach-Lie algebra $\mathcal{B}_{2}(\mathcal{H})_{a h}$ can be decomposed,

$$
\mathcal{B}_{2}(\mathcal{H})_{a h}=\mathcal{G} \oplus \mathcal{F}
$$

for $\mathcal{G}=\left\{x \in \mathcal{B}_{2}(\mathcal{H})_{a h}: x A=A x\right\}$ and $\mathcal{F}=\mathcal{G}^{\perp}$. Let

$$
P_{A}: \mathcal{B}_{2}(\mathcal{H})_{a h} \rightarrow \mathcal{F} \subset \mathcal{B}_{2}(\mathcal{H})_{a h}
$$

be the orthogonal projection. Note that since $\operatorname{ker} \delta_{A}=\mathcal{F}$, then

$$
\left.\delta_{A}\right|_{\mathcal{F}}: \mathcal{F} \rightarrow\left(T \mathcal{O}_{A}\right)_{A}
$$

is a linear bijection. If $\left(T \mathcal{O}_{A}\right)_{A} \subset \mathcal{B}_{2}(\mathcal{H})_{h}$ were closed, then $\left.\delta_{A}\right|_{\mathcal{F}}$ would be an isomorphism between Banach spaces, and therefore there would exist a constant $C_{A}$ such that

$$
\|x A-A x\|_{2} \geq C_{A}\left\|x-P_{A}(x)\right\|_{2} .
$$

Theorem 6.2. Let $A \in \mathcal{B}(\mathcal{H})$ be self-adjoint. Then $\left(T \mathcal{O}_{A}\right)_{A} \subset \mathcal{B}_{2}(\mathcal{H})_{h}$ is closed if and only if the spectrum of $A$ is finite.

Proof. First note that if $\left(T \mathcal{O}_{A}\right)_{A} \subset \mathcal{B}_{2}(\mathcal{H})_{h}$ is closed, then

$$
\left\{x A-A x: x \in \mathcal{B}_{2}(\mathcal{H})\right\} \subset \mathcal{B}_{2}(\mathcal{H})
$$

is also closed. Indeed, since $A$ is self-adjoint, the derivation $\delta_{A}$, which is clearly defined on $\mathcal{B}_{2}(\mathcal{H})$, maps $\mathcal{B}_{2}(\mathcal{H})_{a h}$ into $\mathcal{B}_{2}(\mathcal{H})_{h}$ and $\mathcal{B}_{2}(\mathcal{H})_{h}$ into $\mathcal{B}_{2}(\mathcal{H})_{a h}$. Therefore if $\delta_{A}\left(x_{n}\right) \rightarrow y$ in $\mathcal{B}_{2}(\mathcal{H})$, and one decomposes $x_{n}=x_{n}^{a h}+x_{n}^{h}$ in its hermitian and skew-hermitian parts, then both $\delta_{A}\left(x_{n}^{a h}\right) \in \mathcal{B}_{2}(\mathcal{H})_{h}$ and $\delta_{A}\left(x_{n}^{h}\right) \in \mathcal{B}_{2}(\mathcal{H})_{a h}$ are convergent. The hypothesis that $\delta_{A}\left(\mathcal{B}_{2}(\mathcal{H})_{a h}\right)=\left(T \mathcal{O}_{A}\right)_{A}$ is closed clearly also implies that $\delta_{A}\left(\mathcal{B}_{2}(\mathcal{H})_{h}\right)$ is closed, and our claim follows. We may decompose $\mathcal{H}=\mathcal{H}_{p p} \oplus \mathcal{H}_{c}$ in two orthogonal subspaces which reduce $A$, such that $A_{p p}=$ $\left.A\right|_{\mathcal{H}_{p p}}: \mathcal{H}_{p p} \rightarrow \mathcal{H}_{p p}$ has a dense subset of eigenvalues in its spectrum and $A_{c}=$ $\left.A\right|_{\mathcal{H}_{c}}: \mathcal{H}_{c} \rightarrow \mathcal{H}_{c}$ has no eigenvalues. Since this decomposition reduces $A$, then clearly $\delta_{A_{p p}}\left(\mathcal{B}_{2}\left(\mathcal{H}_{p p}\right)\right)$ and $\delta_{A_{c}}\left(\mathcal{B}_{2}\left(\mathcal{H}_{c}\right)\right)$ are closed, by a similar argument as above.

Let us reason first with $A_{c}$, usually called the continuous spectrum part. Note that $\delta_{A_{c}}$ is injective. If $x \in \mathcal{B}_{2}\left(\mathcal{H}_{c}\right)$ satisfies $\delta_{A_{c}}(x)=0$, then $x$ commutes with $A_{c}$, and thus the real and imaginary parts of $x$ commute with $A_{c}$. This means that there is a nonzero compact self-adjoint operator $y$ which commutes with $A_{c}$. This is clearly not possible: let $p$ be any spectral (finite rank) projection of $y$; then $p$ commutes with $A_{c}$, and therefore $p A_{c} p$, being a finite rank self-adjoint operator, would have an eigenvalue, and therefore $A_{c}$ would have an eigenvalue. It follows that $\delta_{A_{C}}$ is bounded below, and therefore by Fialkow's Theorem 6.1, 
$\sigma_{l}\left(A_{c}\right) \cap \sigma_{r}\left(A_{c}\right)=\emptyset$, which is impossible because for self-adjoint operators, the left and right spectra coincide.

Thus $\mathcal{H}_{c}=\{0\}$, and the spectrum of $A=A_{p p}+0_{\mathcal{H}_{c}}$ has a dense subset of eigenvalues. Suppose that there are infinitely many different nonzero eigenvalues $\left\{\lambda_{n}: n \geq 1\right\}$, ordered such that $\left|\lambda_{1}\right| \geq\left|\lambda_{2}\right| \geq \ldots$ Let $\left\{e_{n}: n \geq 1\right\}$ be an orthonormal set in $\mathcal{H}$, consisting of the corresponding eigenvectors of $A$. These vectors span a subspace $\mathcal{H}_{0}$ which reduces $A$. If we denote it by $A_{0}=\left.A\right|_{\mathcal{H}_{0}}$, it is clear again that $\delta_{A_{0}}\left(\mathcal{B}\left(\mathcal{H}_{0}\right)\right)$ is closed. Thus we may suppose $\mathcal{H}=\mathcal{H}_{0}$. We shall write operators in $\mathcal{H}$ as matrices with respect to this basis. Let us show that $\delta_{A}(\mathcal{H})$ is not closed. With these reductions, it is clear that $\mathcal{F}$ consists of diagonal matrices. Therefore $P_{A}(x)$ consists of leaving the main diagonal of $x$ fixed and replacing all nondiagonal entries of $x$ with zeros. For each $n \geq 1$, consider the $n \times n$ matrix $b_{n}$ with $1 / n$ in all entries, $x_{n}$ the operator on $\mathcal{H}$ with matrix $i b_{n}$ in the main $n \times n$ corner block, and zeros elsewhere. Note that $x_{n}$ is $i$ times a rank one projection, and therefore its 2-norm is 1 . Also note that $\left\|P_{A}\left(x_{n}\right)\right\|_{2}=1 / \sqrt{n} \rightarrow 0$. Therefore

$$
\left\|x_{n}-P_{A}\left(x_{n}\right)\right\|_{2} \rightarrow 1 \text {. }
$$

A straightforward matrix computation shows that $x_{n} A-A x_{n}$ is zero, but on the main $n \times n$ corner block, where it has the matrix with $\frac{1}{n}\left(\lambda_{j}-\lambda_{i}\right)$ at the $i, j$-entry. Therefore

$$
\begin{aligned}
\left\|x_{n} A-A x_{n}\right\|_{2}^{2} & =\frac{1}{n^{2}} \sum_{i, j=1}^{n} \lambda_{j}^{2}+\lambda_{i}^{2}-2 \lambda_{j} \lambda_{i}=\frac{2}{n^{2}}\left\{n \sum_{k=1}^{n} \lambda_{k}^{2}-\left(\sum_{k=1}^{n} \lambda_{k}\right)^{2}\right\} \\
& \leq \frac{2}{n} \sum_{k=1}^{n} \lambda_{k}^{2} \leq \frac{2}{n}\|A\|_{2}^{2}
\end{aligned}
$$

thus $\left\|x_{n} A-A x_{n}\right\|_{2} \rightarrow 0$. It follows that $\left(T \mathcal{O}_{A}\right)_{A}$ is not closed.

If the spectrum of $A$ is finite, then $A=\sum_{i=1}^{n} \lambda_{i} p_{i}$ for pairwise orthogonal selfadjoint projections $p_{i}$ which sum 1 . One can write operators in $\mathcal{B}_{2}(\mathcal{H})$ as $n \times$ $n$ matrices in terms of the decomposition $\mathcal{H}=\sum_{i=1}^{n} R\left(p_{i}\right)$. A straightforward computation shows that if $x \in \mathcal{B}_{2}(\mathcal{H})_{a h}$ with matrix $\left(x_{i, j}\right)$, then $\delta_{A}(x)$ is, in matrix form,

$\delta_{A}(x)=\left(\begin{array}{lllll}0 & \left(\lambda_{2}-\lambda_{1}\right) x_{1,2} & \left(\lambda_{3}-\lambda_{1}\right) x_{1,3} & \ldots & \left(\lambda_{n}-\lambda_{1}\right) x_{1, n} \\ \left(\lambda_{1}-\lambda_{2}\right) x_{2,1} & 0 & \left(\lambda_{3}-\lambda_{2}\right) x_{2,3} & \ldots & \left(\lambda_{n}-\lambda_{2}\right) x_{2, n} \\ \ldots & \ldots & \ldots & \ldots & \ldots \\ \left(\lambda_{1}-\lambda_{n}\right) x_{n, 1} & \left(\lambda_{2}-\lambda_{n}\right) x_{n, 2} & \left(\lambda_{2}-\lambda_{1}\right) x_{1,2} & \ldots & 0\end{array}\right)$.

Since $\lambda_{i} \neq \lambda_{j}$ if $i \neq j$, it follows that $\left\{x A-A x: x \in \mathcal{B}_{2}(\mathcal{H})_{a h}\right\}$ consists of operators in $\mathcal{B}_{2}(\mathcal{H})_{h}$ whose $n \times n$ matrices have zeros on the diagonal, i.e.

$$
\left\{x A-A x: x \in \mathcal{B}_{2}(\mathcal{H})_{a h}\right\}=\left\{z \in \mathcal{B}_{2}(\mathcal{H})_{h}: p_{i} z p_{i}=0, i=1, \ldots, n\right\},
$$

which is clearly closed in $\mathcal{B}_{2}(\mathcal{H})_{h}$.

Remark 6.3. If the spectrum of $A$ is finite, the optimal constant $C_{A}$ can be computed. If $A=\sum_{i=1}^{n} p_{i}$ as above, the set $\left\{x \in \mathcal{B}_{2}(\mathcal{H}): x A=A x\right\}$ consists of block diagonal matrices. Thus

$$
P_{A}(x)=\sum_{i=1}^{n} p_{i} x p_{i}
$$


Using the matrix form of $\delta_{A}(x)$,

$$
\begin{aligned}
\left\|\delta_{A}(x)\right\|_{2}^{2} & =\sum_{i \neq j}\left\|\left(\lambda_{j}-\lambda_{i}\right) x_{i, j}\right\|_{2} \geq \inf _{i \neq j}\left|\lambda_{j}-\lambda_{i}\right|^{2} \sum_{i \neq j}\left\|x_{i, j}\right\|_{2}^{2} \\
& =\inf _{i \neq j}\left|\lambda_{j}-\lambda_{i}\right|^{2}\left\|x-P_{A}(x)\right\|_{2}^{2} .
\end{aligned}
$$

Thus $C_{A}=\inf _{i \neq j}\left|\lambda_{j}-\lambda_{i}\right|^{2}$.

The finite spectrum situation contains interesting cases. For instance, if $A=P$ is a projection with infinite rank and co-rank, the orbit $\mathcal{O}$ equals the connected component of the restricted Hilbert-Schmidt Grassmannian corresponding to the polarization $\mathcal{H}=R(P) \oplus R(P)^{\perp}$ (see [6, 21]) with virtual dimension 0 (i.e. the component containing $P$ ). From the above result it is clear that the finite spectrum condition is necessary for $\mathcal{O}_{A}$ to be a submanifold of $A+\mathcal{B}_{2}(\mathcal{H})_{a h}$ (or a differentiable manifold with the 2-norm topology). In the rest of this section we shall prove that it is also sufficient.

To establish the equivalence between the existence of the submanifold structure for $\mathcal{O}_{A} \subset A+\mathcal{B}_{2}(\mathcal{H})_{a h}$ and the finite spectrum condition, the following general result on homogeneous spaces is useful. A proof can be found in [20].

Lemma 6.4. Let $G$ be a Banach-Lie group acting smoothly on a Banach space $X$. For a fixed $x \in X$, denote by $\pi_{x}: G \rightarrow X$ the smooth map $\pi_{x}(g)=g \cdot x$. Suppose that

(1) $\pi_{x}$ is an open mapping, when regarded as a map from $G$ onto the orbit $\{g \cdot x: g \in G\}$ of $x$ (with the relative topology of $X$ ).

(2) The differential $d\left(\pi_{x}\right)_{1}:(T G)_{1} \rightarrow X$ splits: its kernel and range are closed complemented subspaces.

Then the orbit $\{g \cdot x: g \in G\}$ is a smooth submanifold of $X$, and the map $\pi_{x}: G \rightarrow$ $\{g \cdot x: g \in G\}$ is a smooth submersion.

Theorem 6.5. $\mathcal{O}_{A} \subset A+\mathcal{B}_{2}(\mathcal{H})_{a h}$ is a differentiable submanifold if and only if the spectrum of $A$ is finite.

Proof. The necessary part is clear. Suppose that the spectrum of $A$ is finite, $A=$ $\sum_{i=1}^{n} \lambda_{i} p_{i}$. We shall use Lemma 6.4 above. Note that in our case $G=U_{2}(\mathcal{H})$, $d\left(\pi_{x}\right)_{1}=\delta_{A}$. Its kernel is complemented, and its range is complemented by the previous theorem. Therefore it remains to prove that $\pi_{A}: U_{2}(\mathcal{H}) \rightarrow \mathcal{O}_{A}$ is open, or equivalently, that it has a local continuous cross section defined on a neighborhood of $A \in \mathcal{O}_{A}$. Since the range of $\delta_{A}$ is closed, there exists a constant $C_{A}$ as in (6.1): $\|x A-A x\|_{2} \geq C_{A}\left\|x-P_{A}(x)\right\|_{A}$, for $x \in \mathcal{B}_{2}(\mathcal{H})_{a h}$. Note that $P_{A}$ can be extended to a \|\| -contractive idempotent map, which we shall still call $P_{A}$ :

$$
P_{A}: \mathcal{B}(\mathcal{H}) \rightarrow\{x \in \mathcal{B}(\mathcal{H}): x A=A x\} \subset \mathcal{B}(\mathcal{H}), \quad P_{A}(x)=\sum_{i=1}^{n} p_{i} x p_{i} .
$$

Clearly, $\left.P_{A}\right|_{\mathcal{B}_{2}(\mathcal{H})}$ is the $T r$-orthogonal projection onto the closed subspace $\{x \in$ $\left.\mathcal{B}_{2}(\mathcal{H}): x A=A x\right\}$. Also it is clear that the inequality (6.1) is still valid for $x \in \mathcal{B}_{2}(\mathcal{H})$. Moreover, $P_{A}$ has the following modular property: if $y, z \in\{x \in$ $\mathcal{B}(\mathcal{H}): x A=A x\}$, then $P_{A}(y x z)=y P_{A}(x) z$. Consider the open ball $B=\{b \in$ $\left.\mathcal{O}_{A}:\|b-A\|_{2}<C_{A}\right\}$. We define the following map in $B$ :

$$
\sigma: B \rightarrow U_{2}(\mathcal{H}), \quad \sigma(b)=u \Omega\left(P_{A}\left(u^{*}\right)\right), \text { if } b=u A u^{*},
$$


where $\Omega$ is the unitary part in the polar decomposition of an invertible operator in $G l_{2}(\mathcal{H})(g=\Omega(g)|g|)$. Several facts involving the well definition of $\sigma$ need to be checked. First note that $P_{A}(u)$ lies in $G l_{2}(\mathcal{H})$ : since $b=u A u^{*} \in B$, one has that

$$
C_{A}\left\|u-P_{A}(u)\right\|_{2} \leq\|u A-A u\|_{2}=\left\|u A u^{*}-A\right\|_{2}<C_{A},
$$

i.e. $\left\|1-P_{A}(u) u^{*}\right\|=\left\|u-P_{A}(u)\right\| \leq\left\|u-P_{A}(u)\right\|_{2}<1$, and thus $P_{A}(u)$ is invertible. Moreover, $P_{A}(u)-1=P_{A}(u-1) \in \mathcal{B}_{2}(\mathcal{H})$, and therefore $P_{A}(u) \in G L_{2}(\mathcal{H})$, and thus $\Omega\left(E(u)^{*}\right) \in U_{2}(\mathcal{H})$. Next note that it does not depend on the unitary $u$ performing $b=u A u^{*}$ : if $b=u^{\prime} A u^{\prime *}$ as well, then $u^{\prime}=u v$ for $v A=A v$, and thus

$$
u^{\prime} \Omega\left(P_{A}\left(u^{\prime *}\right)\right)=u v \Omega\left(P_{A}\left(v^{*} u^{*}\right)\right)=u v \Omega\left(v^{*} P_{A}\left(u^{*}\right)\right)=u \Omega\left(P_{A}\left(u^{*}\right)\right) .
$$

Let us prove that $\sigma$ is continuous. It suffices to show that it is continuous at $A$. Suppose that $u_{n} A u_{n}^{*} \rightarrow A$. Then as above, $\left\|u_{n} A-A u_{n}\right\|_{2} \rightarrow 0$, and therefore $\left\|u_{n}-P_{A}\left(u_{n}\right)\right\|_{2} \rightarrow 0$, or equivalently,

$$
\left\|1-u_{n} P_{A}\left(u_{n}^{*}\right)\right\|_{2}=\left\|1-P_{A}\left(u_{n}\right) u_{n}^{*}\right\|_{2}=\left\|u_{n}-P_{A}\left(u_{n}\right)\right\|_{2} \rightarrow 0 .
$$

Therefore (since $\Omega$ is continuous), $\sigma\left(u_{n} A u_{n}^{*}\right)=u_{n} \Omega\left(P_{A}\left(u_{n}^{*}\right)\right)=\Omega\left(u_{n} P_{A}\left(u_{n}^{*}\right)\right) \rightarrow 1$. Finally, $\sigma$ is a cross section: if $b=u A u^{*}$,

$$
\sigma(b) A \sigma(b)^{*}=u \Omega\left(P_{A}\left(u^{*}\right)\right) A \Omega\left(P_{A}\left(u^{*}\right)\right)^{*} u^{*}=u A u^{*},
$$

because the fact that $P_{A}\left(u^{*}\right)$ commutes with $A$ also implies that $\Omega\left(P_{A}\left(u^{*}\right)\right)$ commutes with $A$.

We finish this section by returning to the case of an arbitrary self-adjoint operator $A$. We shall prove that the projection $P_{A}$ verifies that $\left\|P_{A}(x)\right\| \leq\|x\|$.

Proposition 6.6. The projection $P_{A}$ is \|\| -contractive.

Proof. We shall prove this result by giving an alternate construction of $P_{A}$. Let $\Pi$ be a finite partition of the spectrum of $A$ by Borel sets $\left\{\Delta_{1}, \ldots, \Delta_{n(\Pi)}\right\}$. Denote by $p_{i}$ the spectral projection of $A$ corresponding to the set $\Delta_{i}$, and by

$$
E_{\Pi}(x)=\sum_{i=1}^{n(\Pi)} p_{i} x p_{i}, \quad x \in \mathcal{B}_{2}(\mathcal{H}) .
$$

Consider the partial order $\geq$ on finite partitions given by refinement. Then $\left\{E_{\Pi}\right\}$ is a net of contractions acting in the Hilbert space $\mathcal{B}_{2}(\mathcal{H})$. Therefore it has a weak operator convergent subnet, which for simplicity we shall denote again by $\left\{E_{\Pi}\right\}$. Therefore there exists a contraction $F$ acting on $\mathcal{B}_{2}(\mathcal{H})$ such that

$$
\operatorname{Tr}\left(y^{*} E_{\Pi}(x)\right) \rightarrow \operatorname{Tr}\left(y^{*} F(x)\right), \quad \text { for all } x, y \in \mathcal{B}_{2}(\mathcal{H}) .
$$

We claim that $F$ is the orthogonal projection onto $\mathcal{G}_{p}$, i.e. $F=P_{A}$. First note that if $x \in \mathcal{B}_{2}(\mathcal{H})_{a h}$ commutes with $A$, then it commutes with its spectral projections, and therefore

$$
E_{\Pi}(x)=\sum_{i=1}^{n(\Pi)} p_{i} x p_{i}=x \sum_{i=1}^{n(\Pi)} p_{i}=x ;
$$

thus $F(x)=x$. Let $p$ be a spectral projection of $A$. Since the index set $\{\Pi\}$ of the convergent net $\left\{E_{\Pi}\right\}$ is co-final, there exists a partition $\Pi_{0}$ (an index of the net) which is finer than $\{p, 1-p\}$. Therefore for any partition $\Pi=\left\{p_{1}, \ldots, p_{n(\Pi)}\right\} \geq \Pi_{0}$, 
the projections $p_{i}$ are either sub-projections of $p$ or $1-p$, and thus $p p_{i}=p_{i} p$ equals $p_{i}$ or 0 . Then $p E_{\Pi}(x)=\sum_{p p_{i} \neq 0} p_{i} x p_{i}=E_{\Pi}(x) p$. It follows that for any $x$ and $\Pi \geq \Pi_{0}, A$ commutes with $E_{\Pi}(x)$. Then $A$ commutes with $F(x)$. It follows that $F$ is an idempotent operator acting in $\mathcal{B}_{2}(\mathcal{H})_{a h}$ whose range is $\mathcal{G}_{A}$. Apparently, all the operators $E_{\Pi}$ are symmetric with respect to the trace inner product, therefore $F$ is symmetric. Then $F$ is the $T r$-orthogonal projection onto $\mathcal{G}_{A}$, i.e. $F=P_{A}$. This description of $P_{A}$ allows us to prove that it is also \|\| -contractive. Indeed, note that for any fixed $x$, the net of operators $\left\{E_{\Pi}(x)\right\}$ converges to $P_{A}(x)$ in the weak operator topology: if $\xi, \eta \in \mathcal{H}$, denote by $\xi \otimes \eta$ the rank one operator given by $\xi \otimes \eta(\alpha)=\langle\alpha, \eta\rangle \xi$,

$$
\begin{aligned}
\left\langle E_{\Pi}(x) \xi, \eta\right\rangle & =\operatorname{Tr}\left(\left(E_{\Pi}(x) \xi\right) \otimes \eta\right)=\operatorname{Tr}\left(E_{\Pi}(x) \xi \otimes \eta\right) \\
& =-\left\langle\xi \otimes \eta, E_{\pi}(x)\right\rangle \rightarrow-\left\langle\xi \otimes \eta, P_{A}(x)\right\rangle=\left\langle P_{A}(x) \xi, \eta\right\rangle .
\end{aligned}
$$

On the other hand, the operators $E_{\Pi}(x)$ clearly verify $\left\|E_{\Pi}(x)\right\| \leq\|x\|$. Then $\left\|P_{A}(x)\right\| \leq\|x\|$.

\section{Open Problem: GeOdesics JOINING GIVEn EndPOINTS}

In this section we consider the problem of finding a minimal curve in $\mathcal{O}$ joining two given endpoints. First let us remark that the answer is positive, at least locally, for the case $p=2$. In this case $\mathcal{O}$ is a Riemann-Hilbert manifold, and therefore there exists a uniform radius $R>0$ such that any two elements $x_{0}, x_{1} \in \mathcal{O}$ with $d_{2}\left(x_{0}, x_{1}\right)<R$ are joined by a unique minimal geodesic.

For $p \geq 2$, it was shown in [1] that if $\mathcal{O}=\mathcal{O}_{P}=\left\{u P u^{*}: u \in U_{p}(\mathcal{H})\right\}$, with $P$ an infinite self-adjoint projection of $\mathcal{B}(\mathcal{H})$, then any two elements $P_{0}, P_{1}$ of $\mathcal{O}_{P}$ are joined by a minimal geodesic, which is unique if $\left\|P_{0}-P_{1}\right\|<1$.

Let us state the following partial answer to this question.

Proposition 7.1. Suppose that $\mathcal{G}$ is finite dimensional. If $x_{0}, x_{1} \in \mathcal{O}$ satisfy $d_{p}\left(x_{0}, x_{1}\right)<\pi / 4$, then there exists a unique minimal curve joining them, which is of the form $\delta(t)=e^{t z} \cdot x_{0}$, with $z \in \mathcal{G}_{x_{0}}^{\perp_{p}}$.

Proof. Since $d_{p}\left(x_{0}, x_{1}\right)<\pi / 4$, there exists a smooth curve $\gamma(t) \in \mathcal{O}, t \in[0,1]$ such that $\gamma(i)=x_{i}, i=0,1$, and $L(\gamma)<\pi / 4$. Then by Proposition 4.8, there exists a smooth isometric lift $\Gamma(t) \in U_{p}(\mathcal{H})$ with $\Gamma(0)=1, \Gamma(1)=u_{1}$ and $L_{p}(\Gamma)=L(\gamma)$. Note that $u_{1} \cdot x_{0}=x_{1}$. Denote by $d$ the distance

$$
d=d_{p}\left(1, u_{1} G_{x_{0}}\right)=\inf \left\{d_{p}(1, u): w \in u_{1} G_{x_{0}}\right\}
$$

Let $w_{n}$ be a sequence in $u_{1} G_{x_{0}}$ such that $d_{p}\left(1, w_{n}\right) \rightarrow d$. Since $\mathcal{G}$ is finite dimensional, there exists a convergent subsequence, which we still denote by $w_{n}$, $w_{n} \rightarrow w_{0}$. We may also suppose that $d_{p}\left(1, w_{n}\right)<\pi / 4$ for all $n$. Note that $d_{p}\left(1, w_{0}\right) \leq d_{p}\left(1, u_{1}\right)<\pi / 4$. In other words, $w_{0}$ achieves the distance between $q$ and $u_{1} G_{x_{0}}$. By the convexity property of $d_{p}$, it is unique: if $v_{0}$ is another element with $d=d_{p}\left(1, v_{0}\right)$, and $\mu(t)$ is the geodesic joining $w_{0}$ and $v_{0}$, since the map $f_{p}(t)=d_{p}(1, \mu(t))^{p}$ is strictly convex, it follows that $v_{0}=w_{0}$. Clearly there exists $z \in \mathcal{B}_{p}(\mathcal{H})_{a h}$ such that $\|z\|<\pi / 4$ and $\mu(t)=e^{t z}$ is the minimal curve in $U_{p}(\mathcal{H})$ 
joining 1 and $w_{0}$. Then it is apparent that $\delta(t)=e^{t z} \cdot x_{0}$ is the unique minimal curve joining $x_{0}$ and $x_{1}$ in $\mathcal{O}$. As shown before, the fact that $w_{0}$ is a critical point of the distance function implies that $z \in \mathcal{G}_{x_{0}}^{\perp_{p}}$.

\section{REFERENCES}

[1] E. Andruchow, G. Larotonda, Hopf-Rinow theorem in the Sato Grassmannian, J. Funct. Anal. 255 (2008), no. 7, 1692-1712. MR2442079

[2] E. Andruchow, L. Recht, Geometry of unitaries in a finite algebra: variation formulas and convexity, Int. J. Math (to appear).

[3] E. Andruchow, D. Stojanoff, Geometry of unitary orbits, J. Operator Theory 26 (1991), no. 1, 25-41. MR1214918 (94f:46068)

[4] C. Apostol, L. A. Fialkow, D. A. Herrero, D. V. Voiculescu, Approximation of Hilbert space operators. Vol. II, Research Notes in Mathematics, 102, Pitman (Advanced Publishing Program), Boston, MA, 1984. MR735080 (85m:47002)

[5] D. Beltiţă, Smooth homogeneous structures in operator theory, Chapman et Hall/CRC Monographs and Surveys in Pure and Applied Mathematics, 137, Chapman et Hall/CRC, Boca Raton, FL, 2006. MR2188389(2007c:58010)

[6] D. Beltiță, T. S. Ratiu, A. B. Tumpach, The restricted Grassmannian, Banach Lie-Poisson spaces, and coadjoint orbits, J. Functional Analysis 247 (2007), no. 1, 138-168. MR2319757

[7] P. Bona, Some considerations on topologies of infinite dimensional unitary coadjoint orbits, J. Geom. Phys. 51 (2004), no. 2, 256-268. MR2078674 (2005f:58004)

[8] A. L. Carey, Some homogeneous spaces and representations of the Hilbert Lie group $U(\mathcal{H})_{2}$, Rev. Roumaine Math. Pures Appl. 30 (1985), no. 7, 505-520. MR.826232 (87e:22044)

[9] C. Li, An Estimate for Lipschitz Constants of Metric Projections, J. Math. Anal. Appl. 231 (1999), no. 1, 133-141. MR1676721 (99m:41053)

[10] G. Corach, H. Porta and L. Recht, The geometry of spaces of projections in $C^{*}$-algebras, Adv. in Math. 41 (1997), no. 1, 54-76.

[11] C. E. Durán, L. E. Mata-Lorenzo, L. Recht, Natural variational problems in the Grassmann manifold of a $C^{*}$-algebra with trace, Adv. Math. 154 (2000), 196-228. MR.1780098 (2002e:58012)

[12] P. de la Harpe, Classical Banach-Lie Algebras and Banach-Lie Groups of Operators in Hilbert Space, Lecture Notes in Mathematics 285, Springer, Berlin, 1972. MR0476820 (57:16372)

[13] L. A. Fialkow, $A$ note on norm ideals and the operator $X \longrightarrow A X-X B$, Israel J. Math. 32 (1979), no. 4, 331-348. MR.571087 (81g:47046)

[14] D. A. Herrero, Approximation of Hilbert space operators. Vol. 1. Second edition. Pitman Research Notes in Mathematics Series, 224, Longman Scientific \& Technical, Harlow; copublished in the United States with John Wiley \& Sons, Inc., New York, 1989. MR 1088255 (91k:47002)

[15] S. Lang, Differential and Riemannian manifolds, Third edition. Graduate Texts in Mathematics, 160, Springer-Verlag, New York, 1995. MR1335233 (96d:53001)

[16] G. Larotonda, Unitary orbits in a full matrix algebra, Integ. Equat. Oper. Th. 54 (2006), no. 4, 511-523. MR2222981 (2007c:58008)

[17] L. Mata-Lorenzo and L. Recht, Infinite dimensional homogeneous reductive spaces, Acta Cient. Venezolana 43 (1992), no. 2, 76-90. MR1185114 (93j:46052)

[18] L.E. Mata-Lorenzo, L. Recht, Convexity properties of $\operatorname{Tr}\left[\left(a^{*} a\right)^{n}\right]$, Linear Alg. Appl. 315 (2000), 25-38. MR 1774958 (2002h:47116)

[19] H. Porta and L. Recht, Minimality of geodesics in Grassmann manifolds, Proc. Amer. Math. Soc. 100 (1987), no. 3, 464-466. MR891146 (88f:46113)

[20] I. Raeburn, The relationship between a commutative Banach algebra and its maximal ideal space, J. Functional Analysis 25 (1977), no. 4, 366-390. MR0458180 (56:16383)

[21] G. Segal, G. Wilson, Loop groups and equations of KdV type, Inst. Hautes Études Sci. Publ. Math. No. 61 (1985), 5-65. MR783348 (87b:58039)

[22] R. W. Sharpe, Differential geometry. Cartan's generalization of Klein's Erlangen program. With a foreword by S. S. Chern, Graduate Texts in Mathematics 166, Springer-Verlag, New York, 1997. MR1453120(98m:53033) 
[23] B. Simon, Trace ideals and their applications, Second edition. Mathematical Surveys and Monographs 120, American Mathematical Society, Providence, RI, 2005. MR.2154153 (2006f:47086)

Instituto de Ciencias, J. M. Gutierrez 1150, (1613) Los Polvorines, Buenos Aires, Argentina

E-mail address: eandruch@ungs.edu.ar

Instituto de Ciencias, J. M. Gutierrez 1150, (1613) Los Polvorines, Buenos Aires, Argentina

E-mail address: glaroton@ungs.edu.ar

Departamento de Matemática P y A, Universidad Simón Bolívar, Apartado 89000, Caracas 1080A, Venezuela

E-mail address: recht@usb.ve 